\title{
FINITE GROUPS WITH SYLOW 2-SUBGROUPS OF CLASS TWO. II
}

\author{
BY \\ ROBERT GILMAN AND DANIEL GORENSTEIN
}

\begin{abstract}
In this paper we classify finite simple groups whose Sylow 2-subgroups have nilpotence class two.
\end{abstract}

1. Introduction. In Part II of this paper, we complete the proof of our Main Theorem, stated in the Introduction of Part I, which gives the classification of all finite simple groups with Sylow 2-subgroups of nilpotency class 2 .

We have there defined a restricted simple group $G$ with Sylow 2-subgroups of class 2 to be one which satisfies the following properties:

(1) The composition factors of every proper subgroup of $G$ are isomorphic to known simple groups with Sylow 2-subgroups of class at most 2;

(2) A Sylow 2-subgroup of $G$ does not contain a nontrivial strongly closed abelian subgroup and $G$ has 2-rank at least 3.

Furthermore, we have noted that our Main Theorem is a direct consequence of the classification of restricted simple groups with Sylow 2-subgroups of class 2 . The principal result of Part I (Theorem A) asserted that in a restricted simple group with Sylow 2-subgroups of class 2 , every 2-local subgroup is necessarily 2-constrained and has a trivial core. Hence it remains to treat this case.

We shall here prove:

THEOREM B. If $G$ is a restricted simple group with Sylow 2-subgroups of class 2 in which every 2-local subgroup is 2-constrained and has a trivial core, then $G \cong L_{3}\left(2^{n}\right)$ or $\operatorname{PSp}\left(4,2^{n}\right)$ for some $n \geqslant 2$.

Just as the low 2-rank case of Theorem A depended critically on the fact that a Sylow 2-subgroup $S$ of $G$ does not possess a nontrivial abelian subgroup which is strongly closed in $S$ with respect to $G$, so also does our proof of Theorem B. If $J^{*}(S)$ denotes the Thompson subgroup of $S$ (that is, the subgroup of $S$ generated by its elementary abelian subgroups of maximal rank) and if $D$ denotes the Goldschmidt conjugation family for $S$ (the elements of $D$ thus controlling in the sense of Alperin the fusion in $G$ of the elements of $S$ ), it is a direct consequence of the above condition that there exists an element $D$ in $D$

Presented to the Society, January 25, 1973 under the title Simple groups of class two; received by the editors December $21,1973$.

AMS(MOS) subject classifications (1970). Primary $20 \mathrm{D} 05$. 
such that $J^{*}(S) \nsubseteq D$ (otherwise $Z\left(J^{*}(S)\right.$ ) would be a nontrivial strongly closed abelian subgroup of $S$ ).

The precise structure of the 2-local subgroup $N_{G}(D)$ for such an element $D$ of $D$ is then relentlessly analyzed ( $\S 4$ and 5). This analysis depends in a fundamental way upon a number of very precise properties of groups $H$ which act faithfully on a vector space $V$ over $Z_{2}$ and such that the semidirect product $V \cdot H$ has Sylow 2-subgroups of class 2. On the basis of these module-type properties, which are developed in $\S 2$, we are eventually able to force the exact structure of every maximal 2-local subgroup of $G$.

There turn out to be just two possible structures for the maximal 2-local subgroups of $G$. In the first case, a Sylow 2-subgroup of $G$ is isomorphic to that of the group $L_{3}\left(2^{n}\right), n \geqslant 2$, and then $G \cong L_{3}\left(2^{n}\right)$ by a theorem of Collins [1]. On the other hand, in the second case, we prove that $G$ is a split $(B, N)$ pair of rank 2 with Weyl group isomorphic to $D_{8}$ and conclude by a theorem of Fong and Seitz [2] that $G \cong P \operatorname{Sp}\left(4,2^{n}\right), n \geqslant 2$. These two known results are stated explicitly in $\S 3$.

Unfortunately the method of proof of Theorem B depends too heavily on the fact that $G$ has Sylow 2-subgroups of class 2 to be useful for attacking more general situations involving simple groups whose 2-local subgroups are 2-constrained and have trivial cores.

Finally, we remark that $\mathrm{K}$. Gomi of the University of Tokyo, using a somewhat different approach, has obtained an independent proof of our Theorem $\mathrm{B}$.

2. 2-constrained $C_{2}$-groups. In this section we shall establish a number of very detailed properties of 2-constrained $C_{2}$-groups. Primarily we shall be concerned with questions about the action of $L_{2}\left(2^{n}\right)$ on a vector space $V$ over $Z_{2}$. In this connection, if $L_{2}\left(2^{n}\right)=S L\left(2^{n}\right)$ acts on an elementary abelian 2 group $V$, we say that $V$ is a standard or natural module for $L_{2}\left(2^{n}\right)$ if $V$ has rank $2 n$ and can be identified with the additive group of a vector space of dimension 2 over the field $G F\left(2^{n}\right)$ in such a way that the given action of $L_{2}\left(2^{n}\right)$ becomes its natural 2-dimensional one over $G F\left(2^{n}\right)$.

Let then $H$ be a 2-constrained $C_{2}$-group with $O(H)=1$ in which a Sylow 2-subgroup has class 2. Set $D=O_{2}(H)$ and $Z=\Omega_{1}(Z(D))$ and define $\bar{H}=$ $H / C_{H}(Z), \widetilde{H}=H / O(H)$, and $H^{*}=O^{2}(H)$. By [I, (2.1)], we have that $Z(S)$ $\subseteq Z$. We fix this notation for the section. For the sake of clarity, we restate here [I, (2.65)] and [I, (2.66)].

LEMMA 2.1. The following conditions hold:

(i) $\bar{S}$ is elementary abelian;

(ii) $\widetilde{H}^{*}=L(\widetilde{H}) \times O_{2}(\widetilde{H})$ and each component of $L(\widetilde{H})$ is isomorphic to $L_{2}(q), q \equiv 3,5(\bmod 8)$ or $q=2^{n}, J_{1}$, or is of Ree type of characteristic 3; 
(iii) $H^{*}$ acts trivially on $D^{\prime}, \mho^{1}(D)$, and $D / Z$, and $O^{2}\left(O^{2}\left(C_{H}(Z)\right)\right)$ centralizes $D$;

(iv) $C_{H^{*}}(Z)=D$ and $\bar{H}^{*}=H^{*} / D$.

LEMMA 2.2. If $\bar{H} \cong L_{2}\left(2^{n}\right), n \geqslant 1$, and $V$ is a nontrivial $\bar{H}$-composition factor of $Z$, then

(i) $m(V)=2 n$ and $V$ is a standard module for $\bar{H}$;

(ii) For any $\bar{x}$ in $\bar{S}^{\#}, C_{V}(\bar{x})=C_{V}(\bar{S})=[\bar{S}, V]=[\bar{x}, V] \cong E_{2^{n}}$;

(iii) Every element of $N_{\bar{H}}(\bar{S})^{\#}$ of odd order acts regularly on $V$;

(iv) $\left|Z(S): \mho^{1}(D)\right| \geqslant 2^{n}$ and $m(Z) \geqslant 2 n$.

We now prove

LEMma 2.3. Suppose that $\bar{H}^{*}$ is the direct product of subgroups $\bar{H}_{i}, 1 \leqslant$ $i \leqslant t$. If $\bar{H}^{*}$ acts faithfully and irreducibly on an $\bar{H}^{*}$-composition factor of $Z$, then $t=1$.

Proof. Suppose false, so that $t>1$ and $\bar{H}^{*}$ acts faithfully and irreducibly on some $\bar{H}^{*}$-composition factor $V$ of $Z$. Setting $\bar{H}_{2}^{*}$ equal to the product of the $\bar{H}_{i}$ for $2 \leqslant i \leqslant t$, we have that $\bar{H}^{*}=\bar{H}_{1} \times \bar{H}_{2}^{*}$. Hence without loss we can assume that $t=2$. Note that as $\bar{H}^{*}=O^{2^{\prime}}(\bar{H})$, we have that $O^{2^{\prime}}\left(\bar{H}_{i}\right)=$ $\bar{H}_{i}$ and so also $\bar{H}_{i}$ is generated by its 2-elements, $i=1,2$.

Since $\bar{H}_{2}$ centralizes $\bar{H}_{1}$, Clifford's theorem tells us that $V$ is a sum of isomorphic irreducible $\bar{H}_{1}$-modules. Let $W$ be one of these submodules. If $W$ $=V$, then $\bar{H}_{1}$ acts faithfully and irreducibly on $V$, whence $\bar{H}_{2}$ must be cyclic of odd order by Schur's lemma, which is not the case. Thus $W \subset V$. Since $\bar{H}_{1}$ acts faithfully on $V$ and $V$ is a homogeneous $\bar{H}_{1}$-module, $\bar{H}_{1}$ must act faithfully on $W$, whence $[W, \bar{x}] \neq 1$ for any 2-element $\bar{x}$ in $\bar{H}_{1}^{\#}$. But now for any 2-element $\bar{y}$ of $\bar{H}_{2}$, we have $[W, \bar{x}, \bar{y}]=1$ as $S$ has class 2 , whence $\bar{y}$ centralizes $[W, \bar{x}]$ and so $W \cap W^{\bar{y}} \neq 1$. But as $\bar{H}_{1}$ centralizes $\bar{y}, \bar{H}_{1}$ leaves $W \cap W^{\bar{y}}$ invariant, forcing $W^{\bar{y}}=W$. Thus every 2-element of $\bar{H}_{2}$ leaves $W$ invariant and we conclude that $\bar{H}_{2}$ leaves $W$ invariant. Hence $W=V$, which is not the case. Thus $t=1$, as asserted.

LEMMA 2.4. If $\bar{H}$ is a direct product of subgroups $\bar{H}_{i} \cong L_{2}\left(2^{n_{i}}\right), n_{i} \geqslant 1$, $1 \leqslant i \leqslant m$, then

(i) $m(Z) \geqslant 2 m(\bar{H})$ and $m\left(Z \cap S^{\prime}\right) \geqslant m(\bar{H})$;

(ii) If $m(Z)=2 m(\bar{H})$, then $\left[Z, \bar{H}_{i}\right]$ is a standard $\bar{H}_{i}$-module;

(iii) If $m\left(Z / C_{Z}(\bar{S})\right) \leqslant m(\bar{H})$, then $Z=Z_{0} Z_{1} \cdots Z_{m}$, where $Z_{0}=$ $C_{Z}\left(\bar{H}^{\prime}\right), Z_{i}=\left[Z, \bar{H}_{i}^{\prime}\right], 1 \leqslant i \leqslant m$. Furthermore, $\left[Z_{i}, \bar{H}_{j}\right]=1$ if $i \neq j$, $Z_{i} /\left(Z_{i} \cap Z_{0}\right)$ is a standard $\bar{H}_{i}$-module, and $Z_{i} \cap Z_{0} \subseteq\left[Z_{i}, \bar{S} \cap \bar{H}_{i}\right]$. Moreover, $Z_{0} \cap Z_{i}=1$ if $n_{i}=1$. 
Proof. Let $r=m(\bar{H})=\left(n_{1}+\cdots+n_{m}\right)$. By Lemma 2.3 there exist distinct factors $V_{i}$ in an $\bar{H}$-composition series for $Z$ such that $\bar{H}_{i}$ acts nontrivially and irreducibly on $V_{i}$ and $\bar{H}_{j}$ centralizes $V_{i}$ for $i \neq j, 1 \leqslant i, j \leqslant m$. Let $\bar{S}_{i}=\bar{S} \cap \bar{H}_{i}$. If $n_{i}=1$, we certainly have $m\left(V_{i}\right) \geqslant 2 n_{i}$ and $m\left(\left[V, \bar{S}_{i}\right]\right)$ $\geqslant n_{i}$, while if $n_{i} \geqslant 2$, the same result holds by Lemma 2.2. Thus $m(Z) \geqslant 2 r$ and, as $\left[Z, \bar{S}_{i}\right]$ covers $\left[V_{i}, \bar{S}_{i}\right], m\left(Z \cap S^{\prime}\right) \geqslant r$. We have proved (i), and next we claim (iii) implies (ii). Indeed, if $m(Z)=2 r$, then the $V_{i}$ 's are a complete set of composition factors for $Z$ and consequently $Z_{0}=1$. Since $[Z, \bar{S}]$ covers $\left[V_{i}, \bar{S}_{i}\right]$ and $[Z, \bar{S}] \subseteq C_{Z}(\bar{S})$,

$$
\left|Z: C_{Z}(\bar{S})\right| \leqslant|Z:[Z, \bar{S}]| \leqslant \prod_{i=1}^{m}\left|V_{i}:\left[V_{i}, \bar{S}\right]\right|=2^{r}
$$

and so the hypothesis of (iii) holds. But now the conclusion of (iii) together with $Z_{0}=1$ gives (ii).

Finally, we prove (iii) by induction on $m$ for any $\bar{H}$-module $Z$ satisfying our hypothesis. Define $Z_{i}$ as in (iii). Since

$$
\left|Z: C_{Z}(\bar{S})\right| \geqslant \prod_{i=1}^{m}\left|V_{i}: C_{V_{i}}(\bar{S})\right| \geqslant \prod_{i=1}^{m} 2^{n_{i}}=2^{r}
$$

we must have equality throughout. Thus $V_{i}$ is the unique nontrivial composition factor for $\bar{H}_{i}$ and $\left|V_{i}: C_{V_{i}}\left(\bar{S}_{i}\right)\right|=2^{n_{i}}$. Suppose first that some $\bar{H}_{i}$, say $\bar{H}_{1} \cong$ $L_{2}(2)$, and let $\bar{F}=O\left(\bar{H}_{1}\right)$. Then $Z=U \times V$, where $U=[Z, \bar{F}], V=C_{Z}(\bar{F})$, and $U$ and $V$ are $\bar{H}$-invariant. $U$ must be an irreducible $\bar{H}_{1}$-module with $\left|U: C_{U}(\bar{x})\right|=2$ for $\bar{x} \in \bar{S}_{1}^{\#}$. If $\bar{y}$ is a distinct conjugate of $\bar{x}$ in $\bar{H}_{1}$, then $\bar{H}_{1}=\langle\bar{x}, \bar{y}\rangle$ centralizes $C_{U}(\bar{x}) \cap C_{U}(\bar{y})$, whence $|U|=4$ and it is immediate that $U$ is a standard $\bar{H}_{1}$-module. Furthermore, $U=Z_{1}$ and $Z_{0} \subseteq V$ by definition of $Z_{1}$ and $Z_{0}$. Thus $Z_{1} \cap Z_{0}=1$. If $m=1$, we are done, while if $m>1, \bar{H}_{i}$ centralizes $Z_{1}$ for $i>1$ by Lemma 2.3 and it is easy to see that $m\left(V / C_{V}\left(\bar{S}_{2} \bar{S}_{3} \cdots \bar{S}_{m}\right)\right) \leqslant m\left(\bar{S}_{2} \bar{S}_{3} \cdots \bar{S}_{m}\right)$. Now (iii) follows from an application of the induction hypothesis to the group $\bar{H}_{2} \bar{H}_{3} \cdots \bar{H}_{n}$ acting on $V$.

It remains to consider the case in which $n_{i} \geqslant 2$ for all $i, 1 \leqslant i \leqslant m$. Then $\bar{H}_{i}=\bar{H}_{i}^{\prime}$ for all $i$. Set $W_{i}=C_{Z_{i}}\left(\bar{H}_{i}\right), 1 \leqslant i \leqslant m$. As each $\bar{H}_{i}$ has just one nontrivial composition factor, $Z_{i} / W_{i}$ must be a standard $\bar{H}_{i}$-module. Let $\bar{F}_{i}$ be a complement to $\bar{S}_{i}$ in $N_{\bar{H}_{i}}\left(\bar{S}_{i}\right)$. Since $\bar{F}_{i}$ acts trivially on $W_{i}$ and regularly on $Z_{i} / W_{i}$ and $\bar{F}_{i}$ has odd order, we have $Z_{i}=\left[Z_{i}, \bar{F}_{i}\right] \times W_{i}$ with $\bar{F}_{i}$ acting regularly on $\left[Z_{i}, \bar{F}_{i}\right]$. Since $\left[Z_{i}, \bar{S}_{i}\right]$ is $\bar{F}_{i}$-invariant, it is therefore the product of its intersections with the two factors of $Z_{i}$ and consequently $X_{i}$ $=\left[Z_{i}, \bar{F}_{i}\right] \times\left[Z_{i}, \bar{S}_{i}\right] \cap W_{i} \supseteq\left[Z_{i}, \bar{S}_{i}\right]$. This implies that $X_{i}$ is $\bar{S}_{i}$-invariant. But also each factor of $X_{i}$ is clearly invariant under $N_{\bar{H}_{i}}\left(\bar{F}_{i}\right)$. Since $\bar{H}_{i}=$ 
$\left\langle N_{\bar{H}_{i}}\left(\bar{F}_{i}\right), \bar{S}_{i}\right\rangle$ by [I, (2.36)(ii)], it follows now that $X_{i}$ is $\bar{H}_{i}$-invariant. Since $\bar{H}_{i}$ acts nontrivially on $X_{i} \subseteq Z_{i}$ and $\bar{H}_{i}$ has only one nontrivial composition factor, this forces $X_{i}=Z_{i}$. We conclude from this that $\left[Z_{i}, \bar{S}_{i}\right] \cap W_{i}=W_{i}$, whence $W_{i} \subseteq\left[Z_{i}, \bar{S}_{i}\right]$.

But $\bar{S}$ centralizes $\left[Z_{i}, \bar{S}_{i}\right]$ as $S$ has class 2 and so centralizes $W_{i}$. Since $W_{i}$ is $\bar{H}$-invariant, it follows that every 2-element of $\bar{H}$ centralizes $W_{i}$ and hence that $\bar{H}$ centralizes $W_{i}$. Thus $W_{i} \subseteq Z_{0}$ and so $W_{i}=Z_{i} \cap Z_{0}$. Also by Lemma $2.3, \bar{H}_{j}$ centralizes $Z_{i} / W_{i}$ for $j \neq i$ and consequently $\bar{H}_{j}$ centralizes $Z_{i}$.

Finally set $U=Z_{1} Z_{2} \cdots Z_{m}$. To complete the proof of (iii), it remains only to show that $Z=Z_{0} U$. We have that

$$
\left|U: C_{U}(\bar{S})\right| \geqslant \prod_{i=1}^{m}\left|Z_{i}: C_{Z_{i}}\left(\bar{S}_{i}\right)\right| \geqslant \prod_{i=1}^{m} 2^{n_{i}}=2^{r}=m(\bar{H}) .
$$

Hence by the hypothesis of (iii), it follows that $Z=V U$, where $V=C_{Z}(\bar{S})$. Clearly $Z_{0} \subseteq V$ and $V$ is $\bar{F}$-invariant, where $\bar{F}=\bar{F}_{1} \bar{F}_{2} \cdots \bar{F}_{m}$. On the other hand, $\bar{H}$ has no trivial composition factors on $U / U \cap Z_{0}$ and consequently $\bar{F}$ has no nontrivial fixed points on this factor group. We see then that $C_{Z}(\bar{F}) \subseteq V$. Thus $\bar{S}$ centralizes $C_{Z}(\bar{F})$. But $C_{Z}(\bar{F})$ is invariant under $N_{\bar{H}}(\bar{F})$ and so $\left\langle N_{\bar{H}}(\bar{F})\right.$, $\bar{S}\rangle$ leaves $C_{Z}(\bar{F})$ invariant. Since $\bar{H}=\bar{H}_{1} \times \bar{H}_{2} \times \cdots \times \bar{H}_{m}$, we conclude now from [I, (2.36)(ii)] that $\bar{H}$ leaves $C_{Z}(\bar{F})$ invariant. Since $S$ centralizes this group, it follows that $\bar{H}$ does as well and so $C_{Z}(\bar{F}) \subseteq Z_{0}$. But $\bar{F}$ centralizes $Z / U$ as each $\bar{H}_{i}$ centralizes $Z / U$. Since $\bar{F}$ has odd order, we conclude that $Z_{0}$ covers $Z / U$ and so $Z=Z_{0} U$, as asserted.

LEMMA 2.5. Suppose $\bar{S}$ contains a subgroup $\bar{T}$ of rank $n \geqslant 1$ with the following properties:

(a) $\left|Z: C_{Z}(\bar{T})\right| \leqslant 2^{n}$;

(b) If $n \geqslant 2$, then $C_{Z}(\bar{A})=C_{Z}(\bar{T})$ for all $\bar{A}$ of index 2 in $\bar{T}$.

Then if $\bar{N}$ denotes the normal closure of $\bar{T}$ in $\bar{H}^{*}$, we have

(i) If $n=1$, then $\bar{N} \cong L_{2}(2), Z=[Z, \bar{N}] \times C_{Z}(\bar{N})$ and $[Z, \bar{N}]$ is a standard $\bar{N}$-module;

(ii) If $n \geqslant 2$, then $\bar{N}=\bar{N}_{1} \times \cdots \times \bar{N}_{m}$ with $N_{i} \cong L_{2}\left(2^{n_{i}}\right), n_{i} \geqslant 2$, $1 \leqslant i \leqslant m$, and $Z=Z_{0} Z_{1} \cdots Z_{m}$, where $Z_{0}=C_{Z}(\bar{N}), Z_{i} /\left(Z_{i} \cap Z_{0}\right)$ is a standard $\bar{N}_{i}$-module, $Z_{i} \cap Z_{0} \subseteq\left[Z_{i}, \bar{S} \cap \bar{N}_{i}\right]$, and $\left[Z_{i}, \bar{N}_{j}\right]=1$ for $i \neq j$;

(iii) $\bar{T}$ is a Sylow 2-subgroup of $\bar{N}$ and $\left|Z: C_{Z}(\bar{T})\right|=2^{n}$.

Proof. Suppose first that $n=1$, so that $\bar{T}=\langle\bar{x}\rangle$. Since $O_{2}(\bar{H})=1$, we can find a conjugate $\bar{y}$ of $\bar{x}$ such that $[\bar{x}, \bar{y}] \neq 1$. We have that $\bar{Y}=$ $\langle\bar{x}, \bar{y}\rangle$ is a dihedral group; and we can choose $\bar{y}$ so that $O(\bar{Y})$ has index 2 in 
$\bar{Y}$. In particular $O(\bar{Y}) \neq 1$. Setting $E=C_{Z}(\bar{Y})$, our conditions imply $|Z: E|$ \$4. But $O(\bar{Y})$ must act faithfully on $Z / E$, so $|Z: E|=4$ and $O(\bar{Y}) \cong Z_{3}$. Thus $\bar{Y} \cong L_{2}(2)$ and $Z=E \times F$, where $F=[Z, O(\bar{Y})] \cong E_{4}$.

We shall argue that $\bar{Y} \triangleleft \bar{N}$, which will suffice to establish (i). Indeed, assume that this holds. Then if $\bar{P}$ is an arbitrary Sylow 2-subgroup of $\bar{H}^{*}$, we have that $\bar{P}_{1}=\bar{P} \cap \bar{Y}$ is a Sylow 2-subgroup of $\bar{Y}$. Since $\bar{P}$ is abelian, $\bar{P}$ leaves $\bar{P}_{1}$ invariant. But $O(\bar{Y})=\left[\bar{P}_{1}, \bar{N}\right]$ as $\bar{Y} \triangleleft \bar{N}$ and so $\bar{N}=\bar{Y} \times C_{\bar{N}}(\bar{Y})$.

Hence $\bar{P}$ leaves $O(\bar{Y})$ invariant and so normalizes $\bar{Y}$. Since $\bar{P}$ was arbitrary, we conclude that $\bar{H}^{*}$ normalizes $\bar{Y}$, whence $\bar{Y}=\bar{N}$ and (i) holds by Lemma 2.4.

Suppose then that $\bar{Y} \nless \bar{N}$ in which case there exists a conjugate $\bar{w}$ of $\bar{x}$ such that $\bar{Y} \pitchfork \bar{R}=\langle\bar{Y}, \bar{w}\rangle$. Let $E_{0}=C_{E}(\bar{w}), \bar{Q}=C_{\bar{R}}\left(Z / E_{0}\right) . \bar{R}$ acts on $Z / E_{0}$ and $\bar{Q} \subseteq O_{2}(\bar{R})$. However, $\left|Z: E_{0}\right| \leqslant 8$ and so $\bar{R} / \bar{Q}$ is isomorphic to a subgroup of $L_{3}(2)$. Since $\bar{R}$ has abelian Sylow 2-subgroups and the image of $\bar{Y}$ in $L_{3}(2)$ is isomorphic to $L_{2}(2)$, the only possibility is that $\bar{R}=\bar{Y} \bar{Q}$ whence $\bar{R}=O_{2}(\bar{R}) \times \bar{Y}$. Thus $\bar{Y} \triangleleft \bar{R}$, a contradiction.

Clearly (iii) holds when $n=1$, so it remains to treat the case $n \geqslant 2$. We claim in this case that $\bar{T}$ centralizes any $\bar{T}$-invariant subgroup of $\bar{H}$ of odd order. Indeed, let $\bar{Q}$ be such a subgroup. Then $C_{\bar{Q}}(\bar{A})$ normalizes $C_{Z}(\bar{A})=$ $C_{Z}(\bar{T})$ for any $\bar{A}$ of index 2 in $\bar{T}$. But $\bar{Q}=\left\langle C_{\bar{Q}}(A) \mid \bar{A} \in E_{n-1}(\bar{T})\right\rangle$ and so $\bar{Q}$ leaves $V=C_{Z}(\bar{T})$ invariant. Since $[\bar{T}, Z] \subseteq Z(S) \subseteq V$, we have that $\bar{T}$ centralizes both $V$ and $Z / V$, so $[\bar{Q}, \bar{T}]$ does as well. This forces $[\bar{Q}, \bar{T}]=1$ and our assertion is proved.

In particular, our argument yields that $\bar{N}$ centralizes $O\left(\bar{H}^{*}\right)$. The structure of $\widetilde{H}^{*}=(\bar{H} / O(\bar{H}))^{*}=\bar{H}^{*} / O\left(\bar{H}^{*}\right)$ is given by Lemma $2.1(\mathrm{ii})$. Since $\mathrm{O}_{2}\left(\bar{H}^{*}\right)=$ 1 , it is immediate from this that $C_{\bar{H}^{*}}\left(O\left(\bar{H}^{*}\right)\right)=L(\bar{H}) Z\left(O\left(\bar{H}^{*}\right)\right)$. Since $\bar{N}=$ $O^{2}(\bar{N})$ and $\bar{N}$ centralizes $O(\bar{H})$, we conclude that $\bar{N} \subseteq L(\bar{H})$. Since $\bar{N} \triangleleft$ $L(\bar{H})$, this in turn implies that $\bar{N}$ is semisimple. If $\bar{U}$ is any subgroup of $\bar{S} \cap$ $\bar{N}$, then $O\left(C_{\bar{N}}(\bar{U})\right)$ is $\bar{T}$-invariant of odd order, and so is centralized by $\bar{T}$.

From the generational properties listed in [I, (2.35)] and using the fact that $\bar{S} \cap$ $\bar{N}$ is abelian, it follows that $\bar{T}$ centralizes all components of $\bar{N}$ of type $L_{2}(q)$, $q$ odd, $q \neq 5$, or of Ree type. But as $\bar{N}$ is generated by conjugates of $\bar{T}$, clearly $\bar{T}$ centralizes no component of $\bar{N}$. Hence $\bar{N}$ has no components of either type, whence $\bar{N}$ is a product of components $\bar{N}_{i} \cong L_{2}\left(2^{n_{i}}\right), n_{i} \geqslant 2$, or $J_{1}, 1 \leqslant i \leqslant m$.

Let $V_{i}$ be an $\bar{N}$-composition factor of $Z$ on which $\bar{N}_{i}$ acts nontrivially. By Lemma 2.3 , all $\bar{N}_{j}, j \neq i$, act trivially on $V_{i}$ and so $\bar{N}_{i}$ acts faithfully and irreducibly on $V_{i}$. If $\bar{N}_{i} \cong L_{2}\left(2^{n} i\right)$, then by Lemma $2.2, m\left(V_{i}\right)=2 n_{i}$ and 
$\left|V_{i}: C_{V_{i}}\left(\bar{U}_{i}\right)\right|=2^{n_{i}}$ for any nontrivial subgroup $\bar{U}_{i}$ of $\bar{S} \cap \bar{N}_{i}$. We claim that if $\bar{N}_{i} \cong J_{1}$, then, in fact, $\left|V_{i}: C_{V}\left(\bar{U}_{i}\right)\right|>\left|\bar{U}_{i}\right|$ for any such subgroup $\bar{U}_{i}$. Indeed, an element of order 19 in $J_{1}$ is strongly real by [3]. Since $J_{1}$ has only one conjugacy class of involutions, it follows that for $\bar{x}$ in $\bar{U}_{i}^{\#}$, there exists a conjugate $\bar{y}$ of $\bar{x}$ in $\bar{H}_{i}$ such that $\bar{Y}=\langle\bar{x}, \bar{y}\rangle$ is dihedral of order 38 . Hence if we set $E_{i}=C_{V_{i}}(\bar{Y})$, we have that an element of order 19 in $\bar{Y}$ must act faithfully on $V_{i} / E_{i}$ and consequently $m\left(V_{i} / E_{i}\right) \geqslant 18$. On the other hand, $m\left(V_{i} / E_{i}\right) \leqslant 2 m\left(V_{i} / C_{V_{i}}(\bar{x})\right)$, whence $m\left(V_{i} / C_{V_{i}}(\bar{x})\right) \geqslant 9$. Since $\bar{x} \in \bar{U}_{i}^{\#}$ and $m\left(\bar{U}_{i}\right) \leqslant 3$, we conclude from this that $m\left(V_{i} / C_{V_{i}}\left(\bar{U}_{i}\right)\right)>m\left(\bar{U}_{i}\right)$, which gives the desired assertion.

We now take as $\bar{U}_{i}$ the projection of $\bar{T}$ on $\bar{N}_{i}, 1 \leqslant i \leqslant m$. Since $\bar{T}$ centralizes no $\bar{N}_{i}$, each $\bar{U}_{i} \neq 1$. By assumption (a), we have $|\bar{T}| \geqslant\left|Z: C_{Z}(\bar{T})\right|$ and so

$$
|\bar{T}| \geqslant\left|Z: C_{Z}(\bar{T})\right| \geqslant \prod_{i=1}^{m}\left|V_{i}: C_{V_{i}}\left(\bar{U}_{i}\right)\right| \geqslant \prod_{i=1}^{m}\left|\bar{U}_{i}\right| \geqslant|\bar{T}|
$$

Hence all the preceding inequalities are equalities. In particular, our analysis shows that no $\bar{N}_{i} \cong J_{1}$ and so $\bar{N}_{i} \cong L_{2}\left(2^{n}\right), 1 \leqslant i \leqslant m$. Likewise these equalities imply that $\left|\bar{U}_{i}\right|=2^{n_{i}}, 1 \leqslant i \leqslant m$, and that $\bar{T}=\bar{U}_{1} \times \bar{U}_{2} \times \cdots \times \bar{U}_{m}$. Thus $\bar{T}$ projects onto a Sylow 2-subgroup of each $\bar{N}_{i}$ and we conclude that $\bar{T}$ is a Sylow 2-subgroup of $\bar{N}$. In addition, our equalities yield that $\left|Z: C_{Z}(\bar{T})\right|=$ $2^{n}$, so (iii) holds.

Finally by Lemma 2.4 , (ii) holds unless $n_{i}=1$ for some $i$. In this case, the assumption $n \geqslant 2$ forces $m \geqslant 2$. But then if $\bar{A}$ denotes the product of all the $\bar{U}_{j}$ except for $\bar{U}_{i}, 1 \leqslant j \leqslant m$, we have that $|\bar{T}: \bar{A}|=2$ and that $C_{V_{i}}(\bar{A})$ $\neq C_{V_{i}}(\bar{T})$. The preceding analysis then shows that $C_{Z}(\bar{A}) \neq C_{Z}(\bar{T})$, contrary to our hypothesis. Hence this case cannot arise and so (ii) holds.

We shall also need the following variations of Lemma 2.5 .

LEMmA 2.6. Suppose $\bar{S}$ contains a subgroup $\bar{T}$ of rank $n \geqslant 1$ with the following properties:

(a) $\left|Z: C_{Z}(\bar{T})\right| \leqslant 2^{n}$;

(b) $C_{Z}(\bar{t})=C_{Z}(\bar{T})$ for all $\bar{t}$ in $\bar{T}^{\#}$.

Then the normal closure of $\bar{T}$ in $\bar{H}^{*}$ is isomorphic to $L_{2}\left(2^{n}\right)$.

Proof. If $n=1$, (b) automatically holds and the result follows from Lemma 2.5 (i), so we can assume that $n \geqslant 2$. Then by Lemma 2.5 , the normal closure $\bar{N}$ of $\bar{T}$ in $\bar{H}^{*}$ is a direct product of subgroups $\bar{N}_{i} \cong L_{2}\left(2^{n}\right), n_{i} \geqslant 2$, $1 \leqslant i \leqslant m, \bar{T}$ is a Sylow 2-subgroup of $\bar{N}$, and in an $\bar{N}$-composition series for $Z$, 
each $\bar{N}_{i}$ acts nontrivially on $Z_{i}$, where $Z_{i}$ is as in Lemma 2.5 . We need only prove that $m=1$, for then $\bar{T}$ will be a Sylow 2-subgroup of $\bar{N}=\bar{N}_{1}$, forcing $n=n_{1}$, and the desired assertion will hold. We can suppose that $m \geqslant 2$. Let $\bar{x}_{i} \in\left(\bar{T} \cap \bar{N}_{i}\right)^{\#}$, which is possible as $\bar{T}$ is a Sylow 2-subgroup of $\bar{N}$. Since $\bar{N}_{1}$ acts nontrivially on $Z_{1}, Z_{1} \nsubseteq C_{Z}\left(\bar{x}_{1}\right)$. By our hypothesis, $C_{Z}\left(\bar{x}_{1}\right)=C_{Z}\left(\bar{x}_{2}\right)=$ $C_{Z}(\bar{T})$ and so $C_{Z}\left(\bar{x}_{2}\right)$ does not contain $Z_{1}$. However, by Lemma $2.5\left[Z_{1}\right.$, $\left.\bar{N}_{2}\right]=1$, contradicting $m \geqslant 2$.

Lemma 2.7. Suppose $\bar{S}$ contains a subgroup $\bar{T}$ of rank $n \geqslant 1$ with the following property:

(a) $\left|Z: C_{Z}(\bar{T})\right| \leqslant 2^{n}$;

Then if $\bar{N}$ denotes the normal closure of $\bar{T}$ in $\bar{H}^{*}$, we have

(i) $\bar{N}=\bar{N}_{1} \times \cdots \times \bar{N}_{m}$, where $\bar{N}_{i} \cong L_{2}\left(2^{n_{i}}\right), n_{i} \geqslant 1,1 \leqslant i \leqslant m$;

(ii) $Z=Z_{0} Z_{1} \cdots Z_{m}$, where $Z_{0}=C_{Z}(\bar{N}), Z_{i} /\left(Z_{i} \cap Z_{0}\right)$ is a standard $\bar{N}_{i}$ module and $\left[Z_{i}, \bar{N}_{j}\right]=1$ for $i \neq j$;

(iii) $\bar{T}$ is a Sylow 2-subgroup of $\bar{N}$ and $\left|Z: C_{Z}(\bar{T})\right|=2^{n}$.

Proof. By hypothesis, we have $\left|Z: C_{Z}(\bar{T})\right| \leqslant|\bar{T}|$. Choose $\bar{T}_{0} \neq 1$ in $\bar{T}$ minimal subject to the condition $\left|Z: C_{Z}\left(\bar{T}_{0}\right)\right| \leqslant\left|\bar{T}_{0}\right|$. If $m\left(\bar{T}_{0}\right)=1$, Lemma 2.5 is certainly applicable to $\bar{T}_{0}$. We claim that the same holds if $m\left(\bar{T}_{0}\right) \geqslant 2$. Indeed, let $\bar{A}$ be of index 2 in $\bar{T}_{0}$. If $C_{Z}(\bar{A}) \supset C_{Z}\left(\bar{T}_{0}\right)$, then it is immediate that $\left|Z: C_{Z}(\bar{A})\right| \leqslant|\bar{A}|$. But then as $1 \neq \bar{A} \subset \bar{T}_{0}$, our minimal choice of $\bar{T}_{0}$ would be contradicted. Thus $C_{Z}(\bar{A})=C_{Z}\left(\bar{T}_{0}\right)$ for all such $\bar{A}$ and so the assumptions of Lemma 2.5 hold in this case as well. Let $\bar{M}$ be the normal closure of $\bar{T}_{0}$ in $\bar{H}^{*}$ so that $\bar{M}=\bar{M}_{1} \times \bar{M}_{2} \times \cdots \times \bar{M}_{r}$, where $\bar{M}_{i} \cong L_{2}\left(2^{h_{i}}\right)$, $h_{i} \geqslant 1,1 \leqslant i \leqslant r$, and $\bar{T}_{0}$ is a Sylow 2 -subgroup of $\bar{M}$. Since $\bar{T}$ is elementary abelian, it follows from the structure of groups with abelian Sylow 2-subgroups that $\overline{M T}=\bar{M} \times \bar{T}_{1}$, where $\bar{T}_{1}$ is a suitable complement to $\bar{T}_{0}$ in $\bar{T}$. Let $\bar{K}$ be the normal closure of $\bar{T}_{1}$ in $\bar{H}^{*}$. Since $\bar{M} \triangleleft \bar{H}^{*}$, we have that $\bar{M}$ centralizes $\bar{K}$. By the structure of $\bar{M}, \overline{M K}=\bar{M} \times \bar{K}$. Moreover, clearly $\bar{N}=\overline{M K}$.

We can suppose that $\bar{T}_{1} \neq 1$ or else the desired conclusions follow from Lemma 2.5. We shall argue that $\bar{T}_{1}$ satisfies the assumption of the lemma. Let $Z_{0} Z_{1} \cdots Z_{r}$ be the decomposition of $Z$ as an $\bar{M}$-module given by Lemma 2.5 (applied to the inverse image of $\bar{M}$ in $H$ ). As $\left(Z_{i} \cap Z_{0}\right) \subseteq\left[Z_{i}, \bar{S} \cap \bar{M}_{i}\right], \bar{T}_{1}$ centralizes $Z_{i} \cap Z_{0}, 1 \leqslant i \leqslant r$. By Lemma $2.3, \bar{T}_{1}$ centralizes $Z_{i} /\left(Z_{i} \cap Z_{0}\right)$, and it follows that for $\bar{t} \in \bar{T}_{1}$ and $a \in Z_{i}$, the map $a \rightarrow[a, \bar{t}]$ is a homomorphism of $\bar{M}$-modules. We conclude that $\bar{T}_{1}$ centralizes $Z_{i}, 1 \leqslant i \leqslant r$. Since $\bar{T}_{0}$ centralizes $Z_{0}$, we have

$$
\left|Z: C_{Z}(\bar{T})\right|=\left|Z: C_{Z}\left(\bar{T}_{0}\right)\right|\left|Z: C_{Z}\left(\bar{T}_{1}\right)\right| .
$$


On the other hand, by Lemma 2.5 (iii), $\left|Z: C_{Z}\left(\bar{T}_{0}\right)\right|=\left|\bar{T}_{0}\right|$. Hence by our hypothesis, we calculate that

$$
\left|Z: C_{Z}\left(\bar{T}_{1}\right)\right|=\left|Z: C_{Z}(\bar{T})\right|\left|Z: C_{Z}\left(\bar{T}_{0}\right)\right|^{-1} \leqslant|\bar{T}|\left|\bar{T}_{0}\right|^{-1}=\left|\bar{T}_{1}\right| .
$$

We conclude therefore by induction on the order of $|\bar{T}|$ that the lemma holds for $\bar{T}_{1}$. Hence $\bar{K}$ is a direct product of $L_{2}\left(2^{n}\right)$ 's and as $\bar{N}=\bar{M} \times \bar{K}$, it follows that $\bar{N}$ is as well. Thus (i) holds. Also $\bar{T}$ must be a Sylow 2-group of $\bar{N}$ since $\bar{T}_{0}$ and $\bar{T}_{1}$ are Sylow 2-groups of $\bar{M}$ and $\bar{K}$, respectively. Now (ii) holds by Lemma 2.4, and the last assertion of (iii) follows from our calculation of $\left|Z: C_{Z}(\bar{T})\right|$ above.

LEMMA 2.8. If $\widetilde{H} \cong L_{2}\left(2^{n}\right), n \geqslant 1$, and $\bar{S}$ contains a nontrivial subgroup $\bar{T}$ such that $\left|Z: C_{Z}(\bar{T})\right| \leqslant|\bar{T}|$, then we have

(i) $\bar{H}^{*} \cong L_{2}\left(2^{n}\right)$ and $\bar{T}=\bar{S}$;

(ii) $\left|Z: C_{Z}(\bar{T})\right|=2^{n}$;

(iii) $C_{Z}(\bar{t})=C_{Z}(\bar{T})$ for any $\bar{t}$ in $\bar{T}^{\#}$; and

(iv) $Z=Z_{0} Z_{1}$, where $Z_{0}=C_{Z}\left(\bar{H}^{*}\right)$ and $Z_{1}=\left[Z, \bar{H}^{*}\right]$, and $Z_{1} /\left(Z_{1} \cap Z_{0}\right)$ is a standard $\bar{H}^{*}$-module.

Proof. $\bar{T}$ satisfies the assumptions of Lemma 2.7. Since $\widetilde{H} \cong L_{2}\left(2^{n}\right)$, Lemma 2.7 immediately implies (i), (ii), and (iv). Since $Z / Z_{0}=V$ is a standard $\bar{H}^{*}$-module, (iii) will follow once we show that $C_{Z}(\bar{T})$ covers $C_{V}(\bar{T})$. However, $C_{Z}(\bar{T}) \supseteq[Z, \bar{T}]$, which covers $[V, \bar{T}]=C_{V}(\bar{T})$.

Lemma 2.9. Suppose that $\bar{H} \cong G L\left(2,2^{n}\right), n \geqslant 2$, and that the following conditions hold:

(a) In an $L(\bar{H})$-composition series of $Z, L(\bar{H})$ acts nontrivially on exactly one composition factor and the rank of this composition factor is $2 n$;

(b) $Z=[Z, L(\bar{H})]$;

(c) If $X$ is a complement to $\bar{S}$ in $N_{\bar{H}}(\bar{S})$, then $C_{Z}(\bar{X})=1$. Under these conditions, we have:

(i) $m(Z)=2 n$ or $3 n$ and correspondingly $m(Z(S))=n$ or $2 n$;

(ii) If $Z_{0}=C_{Z}(L(\bar{H}))$, then correspondingly $Z_{0}=1$ and all involutions of $Z$ are conjugate in $H$ or $m\left(Z_{0}\right)=n$ and $Z-Z_{0}$ has two classes of involutions in $H$;

(iii) $S^{\prime}=Z(S)$;

(iv) All involutions of $Z$ are central.

Proof. Let $Z_{1}$ be a maximal $L(\bar{H})$ submodule of $Z$. Since $Z=$ $[Z, L(\bar{H})], L(\bar{H})$ acts faithfully and irreducibly on $Z / Z_{1}$. But then by (a), $L(\bar{H})$ must act trivially on $Z_{1}$ and so $Z_{1}=Z_{0}$. Since $\bar{S} \subseteq L(\bar{H})$, also $Z_{0} \subseteq$ 
$Z(S)$. Now Lemma 2.2 implies that $m\left(Z / Z_{0}\right)=2 n$ and $Z / Z_{0}$ is a standard $L(\bar{H})$-module. Hence $[Z, \bar{S}]$ covers $C_{Z / Z_{0}}(\bar{S})=C_{Z / Z_{0}}(\bar{x}), \bar{x} \in \bar{S}^{\#}$. Since $Z_{0} \subseteq Z(S)$, it follows that $Z(S)=C_{Z}(\bar{S})=C_{Z}(\bar{x})$ and $\left|Z: C_{Z}(\bar{S})\right|=$ $\left|Z: C_{Z}(\bar{x})\right|=2^{n}$. Furthermore, as $Z=[Z, L(\bar{H})]$, Lemma 2.4 (iii) implies that $Z_{0} \subseteq[Z, \bar{S}]$. We see then that $Z(S)=[Z, \bar{S}]$ and consequently $Z(S)=S^{\prime}$, so (iii) holds.

Observe next that by $[\mathrm{I},(2.36)], L(\bar{H})$ is generated by three conjugates $\bar{x}_{1}=\bar{x}, \bar{x}_{2}, \bar{x}_{3}$ of $\bar{x}$. If $U_{i}$ is a complement to $C_{Z}\left(\bar{x}_{i}\right)$ in $Z$, then $\left|U_{i}\right|=$ $2^{n}$ as $\left|Z: C_{Z}\left(\bar{x}_{i}\right)\right|=2^{n}, 1 \leqslant i \leqslant 3$. But $\bar{x}_{i}$ leaves invariant every subgroup of $Z$ containing $U_{i}, 1 \leqslant i \leqslant 3$, and consequently $L(\bar{H})=\left\langle\bar{x}_{1}, \bar{x}_{2}, \bar{x}_{3}\right\rangle$ leaves $U=U_{1} U_{2} U_{3}$ invariant and $m(U) \leqslant 3 n$. But $\bar{x}_{i}$ centralizes $Z / U_{i}$ for each $i$ and so $L(\bar{H})$ acts trivially on $Z / U$. Now condition (b) forces $Z=U$ and we conclude that $m(Z) \leqslant 3 n$.

We now set $W=C_{\bar{H}}(L(\bar{H}))$, so that $\bar{W}$ is cyclic of order $2^{n}-1$ and $\bar{W}$ is a direct factor of $\bar{X}$. In fact, $\bar{X}=\bar{W} \times \bar{X}_{1}$, where $\bar{X}_{1}=\bar{X} \cap L(\bar{H})$ is also cyclic of order $2^{n}-1$. Let $Z_{2}$ be an $\bar{X}$-invariant complement to $Z_{0}$ in $Z$. Then $Z_{2}$ is isomorphic to $Z / Z_{0}$ as a $\bar{W}$-module and so $Z_{2}$ is a homogeneous $\bar{W}$-module. Let $U_{2}$ be the sum of all irreducible $\bar{W}$-submodules of $Z$ that are isomorphic to an irreducible $\bar{W}$-submodule of $Z_{2}$. Since $\bar{W}$ centralizes $L(\bar{H})$, $L(\bar{H})$ leaves $U_{2}$ invariant. But $Z_{2} \subseteq U_{2}$ and so $U_{2}$ covers $Z / Z_{0}$, whence $L(\bar{H})$ acts nontrivially on $U_{2}$. Hence $L(\bar{H})$ acts trivially on $Z / U_{2}$ and again (b) forces $U_{2}=Z$. Thus $Z$ is a homogeneous $\bar{W}$-module. In particular, as $\bar{W}$ acts faithfully on $Z, Z_{0}=1$ or $Z_{0}$ is a faithful $\bar{W}$-module. Since $\bar{W}$ is cyclic of order $2^{n}-1, m\left(Z_{0}\right) \geqslant n$ in the latter case. Thus $m(Z)=2 n$ or $3 n$ and as $|Z: Z(S)|=2^{n}$, we see that (i) holds.

Observe next that $Z(S) \supseteq Z_{0}$ and projects nontrivially in $Z / Z_{0}$. Since $L(\bar{H})$ acts transitively on $\left(Z / \bar{Z}_{0}\right)^{\#}$, it follows at once that all involutions of $Z$ are central in $H$. In particular, (iv) holds. (ii) is clear if $Z_{0}=1$, so suppose $Z_{0} \neq 1$. In view of (iv), (ii) will follow in this case provided we show that $Z(S)$ $-Z_{0}$ has two conjugacy classes of involutions under the action of $\bar{X}$ (since all conjugacy of elements of $Z(S)$ can be realized in $N_{H}(S)$ ). But we know how $\bar{X}$ acts on $Z(S): Z_{0}$ and $U_{2}=Z(S) \cap Z_{2}$ are nonisomorphic $\bar{X}$-modules and $\bar{X}$ acts transitively on both $Z_{0}^{\#}$ and $U_{2}^{\#}$, whence $Z_{0}$ and $U_{2}$ are the only two irreducible $\bar{X}$-submodules of $Z(S)$. Since $\bar{X}$ is abelian, $C_{Z(S)}(\bar{y})$ is an $\bar{X}$-submodule of $Z(S)$ for any $\bar{y}$ in $\bar{X}^{\#}$. It follows at once that $\bar{y}$ has no fixed points on $J=Z(S)-\left(Z_{0} \cup U_{2}\right)$ for any $\bar{y}$ in $\bar{X}^{\#}$. Since $|\bar{X}|=|J|$, we conclude that $\bar{X}$ acts transitively on $J$, so $Z(S)-Z_{0}$ has just two classes of involutions under $X$ and (ii) is proved.

3. Two classification theorems. The proof of Theorem B will also require known characterizations of the groups $L_{3}\left(2^{n}\right)$ and $\operatorname{PSp}\left(4,2^{n}\right)$. 
First of all, the main result of Collins [1] asserts:

Proposition 3.1. If $G$ is a simple group whose Sylow 2-subgroups are isomorphic to those of the group $L_{3}\left(2^{n}\right)$ for some $n \geqslant 2$, then $G \cong L_{3}\left(2^{n}\right)$.

Furthermore, Fong and Seitz [2] have classified all split $(B, N)$-pairs of rank 2 (see [2] for the definition of these terms). A special case of their classification theorem yields the following result:

Proposition 3.2. If $G$ is a split $(B, N)$-pair of rank 2 with Weyl group dihedral of order 8 and with $B \cong H U$, where $U$ is a 2-group of class 2 and order $2^{4 n}$ and $H \cong Z_{2^{n}-1}$ for some $n \geqslant 2$, then $G \cong P S p\left(4,2^{n}\right)$.

4. A characterization of the groups $L_{3}\left(2^{n}\right)$. Henceforth $G$ will denote a simple group which satisfies the hypotheses of Theorem B. Thus $G$ is of restricted type with Sylow 2-subgroup $S$ of class 2 and every 2-local subgroup of $G$ is 2-constrained and has a trivial core.

We denote the Thompson subgroup of $S$ by $J^{*}(S)$. Thus we have

$$
J^{*}(S)=\langle A \mid A \in M(S)\rangle .
$$

Our analysis begins with embedding of $J^{*}(S)$ in the elements of the Goldschmidt conjugation family $D$, which is described in $[I,(2.70)]$. Under our present assumptions on the 2-local subgroups of $G$, we can define $D$ to be the set of nontrivial normal subgroups $D$ of $S$ such that:

(a) $D=O_{2}\left(N_{G}(D)\right)$ and

(b) If $\left.N=N_{G}(D)\right)$ and $\bar{N}=N / D$, then either $|\bar{S}| \leqslant 2$ or $\bar{N} / O(\bar{N})$ contains a normal subgroup of odd index isomorphic to $L_{2}\left(2^{n}\right)$ for some $n \geqslant 2$.

By [I, (2.70)], $D$ is a weak conjugation family for $S$ in the sense of Alperin.

The following result is critical for the proof of Theorem B.

Lemma 4.1. There exists an element $D$ in $D$ such that $J^{*}(S) \nsubseteq D$.

Proof. If false, then $N_{G}(D) \subseteq M=N_{G}\left(J^{*}(S)\right)$ for all $D$ in $D$. Since $D$ is a weak conjugation family, it follows that $M$ controls fusion in $S$. But $Z\left(J^{*}(S)\right)$ is characteristic in $M$ and so is a nontrivial abelian strongly closed subgroup of $S$, contrary to the fact that $G$ is of restricted type.

We choose $D$ in $D$ so that $J^{*}(S) \nsubseteq \subseteq D$; we set $N=N_{G}(D), \bar{N}=N / D$, and $Z=\Omega_{1}(Z(D))$. Thus $O(N)=1, D=O_{2}(N)$, and $N$ is 2-constrained, so that we can apply Lemma 2.1 to $N$ and $\bar{N}$. Since $J^{*}(S) \nsubseteq D$, we can choose $A$ in $M(S)$ with $A \nsubseteq D$. We set $A_{0}=A \cap D$ and we let $W$ be a complement to $A_{0}$ in $A$. As usual, we put $N^{*}=O^{2}(N)$. In fact, throughout the 
balance of Part II it will be convenient to write $X^{*}$ for $O^{2^{\prime}}(X)$ for any group $X$. Finally let $Y_{0}=C_{Z}\left(\bar{N}^{*}\right), B=\left[Z, \bar{N}^{*}\right]=\left[B, \bar{N}^{*}\right]$ and $B_{0}=B \cap Y_{0}$. Note that $Y_{0} \subseteq Z(S)$.

We next prove

LEMMA 4.2. The following conditions hold:

(i) $\bar{N}^{*} \cong L_{2}\left(2^{n}\right)$ for some $n \geqslant 1$;

(ii) $W$ is a complement to $D$ in $S$ and $m(W)=n$;

(iii) $\left|Z: C_{Z}(W)\right|=2^{n}$;

(iv) $C_{Z}(w)=C_{Z}(W)$ for $w$ in $W^{\#}$;

(v) $Z=Y_{0} B$ and $B / B_{0}$ is a standard $\bar{N}^{*}$-module;

(vi) if $n=1$, then $B_{0}=1$ and $B=\left[Z, O^{2}\left(\bar{N}^{*}\right)\right]$;

(vii) $N^{*}$ centralizes $D / Z$.

Proof. We set $m(W)=k$, so that $k \geqslant 1$ and $m\left(A_{0}\right)=m(A)-k$. By [I, (2.70)] either $\bar{N}$ is solvable and $|\bar{S}|=2$ or $\bar{N}^{*} / O\left(\bar{N}^{*}\right) \cong L_{2}\left(2^{n}\right)$ for some $n \geqslant 2$. In the first case we put $n=1$. Then in all cases we have $k \leqslant n$ with equality obviously holding if $n=1$.

Set $Z_{0}=A_{0} \cap Z$ and let $Z_{1}$ be a complement to $Z_{0}$ in $Z$. Since $A \in M(S)$ and $A \cap Z_{1}=1$, it is immediate that $Z_{0}=C_{Z}(A)=C_{Z}(W)$. We also have that $A_{0} Z_{1} \in E(S)$ and consequently

$$
\begin{aligned}
m(S) & =m(A) \geqslant m\left(A_{0} Z_{1}\right) \\
& =m\left(A_{0}\right)+m\left(Z_{1}\right)=m(A)-k+m\left(Z_{1}\right),
\end{aligned}
$$

thus forcing $m\left(Z_{1}\right) \leqslant k$. We conclude that $\left|Z: Z_{0}\right| \leqslant 2^{k}$ and so (i)-(v) hold by Lemma 2.8. Part (vi) follows from Lemma 2.4(iii) and (vii) from Lemma 2.1.

If $n \geqslant 2$, then $\bar{N}^{*}$ is simple, and we may define $J$ to be the unique minimal normal subgroup of $N^{*}$ covering $\bar{N}^{*}$. If $n=1$, define $J=O^{2}\left(N^{*}\right)$; then $J$ is the unique minimal normal subgroup of $N^{*}$ covering $O\left(\bar{N}^{*}\right)$.

LEMMA 4.3. We have

(i) $J \cap D \subseteq Z$;

(ii) $J$ centralizes $D / B$;

(iii) $B=\left[O_{2}(J), J\right]=[B, J]$

(iv) $J$ acts indecomposably on $B$.

Proof. Let $\tilde{N}^{*}=N^{*} / Z ; \widetilde{N}^{*}$ has abelian Sylow 2-subgroups. If $n \geqslant 2$, then $\widetilde{J} \cap \widetilde{D}=1$ by the structure of groups with abelian Sylow 2-subgroups. If $n=1$, then $\widetilde{D} \subseteq Z\left(\tilde{N}^{*}\right)$ by Lemma 4.2 (vii) and it follows easily from the structure of $\bar{N}^{*}$ that $O^{2}\left(\widetilde{N}^{*}\right) \cap \widetilde{D}=1$. Thus (i) holds in both cases. By Lemma 4.2(vii), $J$ centralizes $D / Z$. Since $J$ also centralizes $Z / B$ and $J=$ 
$O^{2}(J)$, it follows that $J$ centralizes $D / B$ and (ii) holds.

Observe next that if $n \geqslant 2$, then $N^{*}=J D$ implies $B=[Z, J]$; and because $J$ is perfect, the three subgroup lemma yields $B=[B, J] \subseteq J$. On the other hand, as $O_{2}(J)=D \cap J$ in all cases, we have, using (i), that $B \subseteq O_{2}(J) \subseteq Z$. Hence if $n \geqslant 2$, we conclude that (iii) holds. In the case $n=1$ we use Lemma 4.2 (vi) and the fact that $\left|J / C_{J}(Z)\right|=|J / J \cap D|$ is odd to obtain $[Z, J]=B=[B, J] \subseteq J$, and we finish the proof of (iii) as in the case $n \geqslant 2$.

Finally if $J$ acted decomposably on $B$, it would follow from Lemma 4.2(v) and the definition of $J$ that $J$ centralized one of the two factors of $B$, contrary to the fact that $B=[B, J]$ by (iii).

We begin to analyze the 2-local subgroups of $G$.

LEMmA 4.4. If $H$ is a 2-local subgroup of $G$ containing $N^{*}$, then $J \triangleleft$ $H^{*}$ and $H^{*} \subseteq N_{G}(B)$.

Proof. Since $B=\left[O_{2}(J), J\right]$ is characteristic in $J$, the first assertion will imply the second.

We set $P=O_{2}(H), \bar{H}=H / P, Y=\Omega_{1}(Z(P))$. Since $S \subseteq N^{*} \subseteq H, S \in$ $S(H)$ and so $P \subseteq S$. But then $P \subseteq O_{2}\left(N^{*}\right)=D$. In particular, $Z \subseteq C_{S}(P) \subseteq$ $P$ and so $Z \subseteq Y$. Furthermore, since $A \in M(G), m(A) \geqslant m(Y(A \cap P))$, whence

$$
\left|Y: C_{Y}(\bar{A})\right| \leqslant|Y: Y \cap A| \leqslant|A: A \cap P|
$$

and Lemma 2.7 implies that $\bar{L}$, the normal closure of $\bar{A}$ in $\bar{H}^{*}$, is a direct product of $L_{2}\left(2^{i}\right)$ 's. By Lemma $4.2, \bar{A}$ covers a Sylow 2-subgroup of $\bar{N}^{*} / \bar{D}$, and it follows that $\bar{L}$ covers $\bar{N}^{*} / \bar{D}$. By the definition of $J$, we have $\bar{J} \subseteq \bar{L}$. Again by Lemma $2.7, \bar{A} \in S(\bar{L})$ and as $A \subseteq N^{*}, \bar{J}$ is $\bar{A}$-invariant.

We claim $\bar{J}$ is a summand of $\bar{L}$ when $n \geqslant 2$, and $\bar{J}$ is the core of a summand of $\bar{L}$ when $n=1$. Suppose $n \geqslant 2$. As $\bar{J}$ is $\bar{A}$-invariant, the projection of $\bar{J}$ on any summand, $\bar{L}_{1}$, of $\bar{L}$ is $\bar{A} \cap \bar{L}_{1}$-invariant. By [I, (2.25)] the projection of $\bar{J}$ on $\bar{L}_{1}$ must then be all of $\bar{L}_{1}$ or trivial. For some choice of $\bar{L}_{1}, \bar{J}$ projects onto $\bar{L}_{1}$, and if $\bar{J}$ projected onto another summand $\bar{L}_{2}, \bar{A}$ $\cap \bar{L}_{1}$ would not normalize $\bar{J}$. Thus $\bar{J}=\bar{L}_{1}$. If $n=1$, then the projection of $\bar{J}$ on $\bar{L}_{1}$ is $\bar{A} \cap \bar{L}_{1}$-invariant of odd order. By [I, (2.33)] it follows that $\bar{J}$ projects trivially on all factors isomorphic to $L_{2}\left(2^{m}\right), m \geqslant 2$. Thus if $\bar{J}$ projects nontrivially on a summand $\bar{L}_{1}$, then $\bar{L}_{1} \cong L_{2}(2)$ and $\bar{J}$ projects onto $O\left(\bar{L}_{1}\right)$. Certainly for some choice of $\bar{L}_{1}, \bar{J}$ does project onto $O\left(\bar{L}_{1}\right)$, and if $\bar{J}$ projected onto $O\left(\bar{L}_{2}\right)$ for some other summand $\bar{L}_{2}$, then $\bar{A} \cap \bar{L}_{1}$ would not normalize $\bar{J}$. We conclude $\bar{J}=O\left(\bar{L}_{1}\right)$, and our claim is proved.

As $\bar{H}$ has abelian Sylow 2-subgroups, it follows from the embedding of $\bar{J}$ in $\bar{L}$ that $\bar{J} \triangleleft \bar{H}^{*}$. Consequently, $J P \triangleleft H^{*}$. But from the definition of $J, J$ is characteristic in $J P$, whence $J \triangleleft H^{*}$. 
Lemma 4.5. There exists an element $D_{1}$ in $D$ such that

(i) $B \nsubseteq D_{1}$ and

(ii) $J^{*}(S) \subseteq D_{1}$.

Proof. From Lemma 4.2, we see that

$|(A \cap D) Z|=\left|Z: C_{Z}(W)\right||A \cap D|=2^{n}|A \cap D|=|W||A \cap D|=|A|$.

Thus $A_{1}=(A \cap D) Z$ lies in $M(S)$, and $B \subseteq Z \subseteq A_{1}$. Hence if $B \subseteq D_{1}$ for some $D_{1}$ in $D$, then $A_{1} \subseteq D_{1}$ and so $J^{*}(S) \subseteq D_{1}$. Therefore, (i) implies (ii). Suppose then that $B \subseteq D_{1}$ for every choice of $D_{1} \in D$. We will obtain a contradiction by producing a nontrivial subgroup $P$ of $S$ such that $N_{G}\left(D_{1}\right) \subseteq$ $N_{G}(P)$ for all choices of $D_{1}$. It will then follow that $N_{G}(P)$ controls fusion in $S$, whence $Z(P)$ is strongly closed in $S$, thus contradicting the fact that $G$ is a restricted simple group.

First, since $B / B_{0}$ is a standard $\bar{N}^{*}$-module, $C_{S}(B) \subseteq D$, whence $B \subseteq D_{1}$ implies that $Z_{1}=\Omega_{1}\left(Z\left(D_{1}\right)\right) \subseteq D$. Set $N_{1}=N_{G}\left(D_{1}\right)$; then by Lemma 2.1, $N_{1}^{*}$ centralizes $D_{1} / Z_{1}$, whence $N_{1}^{*} \subseteq N_{G}\left(D \cap D_{1}\right)$. Likewise $Y_{0} \subseteq Z(S)$ and $Z=$ $B Y_{0}$, so $Z \subseteq B Z(S) \subseteq D_{1}$ and consequently also $N^{*} \subseteq N_{G}\left(D \cap D_{1}\right)$. Thus by Lemma 4.4, we have $N_{1}^{*} \subseteq N_{G}\left(D \cap D_{1}\right)^{*} \subseteq N_{G}(B)$.

On the other hand, as noted in [I, Section 2], $D$ is closed under conjugation by elements of $N_{G}(S)$. Hence by our assumption $B \subseteq D_{1}^{x^{-1}}$ for all $x \in$ $N_{G}(S)$, whence $B^{x} \subseteq D_{1}$ for all $D_{1} \in \mathcal{D}, x \in N_{G}(S)$. Furthermore, if $J^{*}(S)$ $\nsubseteq D$, then also $J^{*}(S) \nsubseteq D^{x}$. In addition, $Z^{x}=\Omega_{1}\left(Z\left(D^{x}\right)\right)$ and $B^{x}=$ $\left[Z^{x},\left(N^{x}\right)^{*}\right]$. We see then that all our analysis applies with $D^{x}$ and $B^{x}$ in the roles of $D$ and $B$. In particular, the conclusion of the preceding paragraph holds for $B^{x}$ and consequently $N_{1}^{*} \subseteq N_{G}\left(B^{x}\right)$ for all $x$ in $N_{G}(S)$. Hence if we set $P=\left\langle B^{x} \mid x \in N_{G}(S)\right\rangle$, we have $1 \neq P \subseteq S$ and $N_{G}(S) \subseteq N_{G}(P)$. Since $N_{1}=N_{1}^{*} N_{N_{1}}(S)$, we conclude now that $N_{1}=N_{G}\left(D_{1}\right) \subseteq N_{G}(P)$. Since this holds for each $D_{1}$ in $D, P$ has the required properties and the lemma is proved.

We remark that Lemmas $4.2-4.4$ hold for any $D_{1} \in D$ with $J^{*}(S) \subseteq D_{1}$. For any such $D_{1}$, denote by $B_{1}, Z_{1}, N_{1}$, and $\bar{N}_{1}$ the subgroups of $G$ corresponding to $B, Z, N$, and $\bar{N}$ respectively.

Lemma 4.6. For any $D_{1}$ satisfying the conditions of Lemma 4.5, we have

(i) $S=B D_{1}=B_{1} D$;

(ii) $\bar{N}^{*} \cong \bar{N}_{1}^{*} \cong L_{2}\left(2^{n}\right)$;

(iii) $B \cap D_{1}=B \cap Z(S)$ and $B_{1} \cap D=B \cap Z(S)$; and

(iv) $D=\left(D \cap D_{1}\right) B$ and $D_{1}=\left(D \cap D_{1}\right) B_{1}$.

Proof. As $B / B_{0}$ is a standard $\bar{N}^{*}$-module, $C_{B}(Q)=B \cap Z(S)$ for any 
subgroup $Q \subseteq S$ with $Q \nsubseteq D$. Likewise $C_{B_{1}}\left(Q_{1}\right)=B_{1} \cap Z(S)$ for any $Q_{1} \subseteq S$ with $Q_{1} \nsubseteq D_{1}$. In particular, $D=C_{S}(B)$ and $D_{1}=C_{S}\left(B_{1}\right)$. As $B \nsubseteq D_{1}$, $\left[B, B_{1}\right] \neq 1$, whence $B_{1} \subseteq D$ and the situation is symmetric. Consequently, $B \cap D_{1}=C_{B}\left(B_{1}\right)=B \cap Z(S)$ and $B_{1} \cap D=C_{B_{1}}(B)=B_{1} \cap Z(S)$. Thus (iii) holds.

Since $|B: B \cap Z(S)|=2^{n}=|S: D|$, we have $\left|S: D_{1}\right| \geqslant\left|B: B \cap D_{1}\right|=$ $|S: D|$. But now by symmetry, $|S: D| \geqslant\left|S: D_{1}\right|$ and so $2^{n}=|S: D|=\left|S: D_{1}\right|$. Lemma 4.2 now yields (ii). Furthermore, the preceding relations imply that $\left|S: D_{1}\right|$ $=\left|B: B \cap D_{1}\right|$, whence $S=B D_{1}$. Again by symmetry, we have $S=B_{1} D$ and so (i) also holds. Finally as $S=B D_{1}, D \subseteq B D_{1}$, whence $D=B\left(D \cap D_{1}\right)$. Since also $S=B_{1} D$, we similarly obtain $D_{1}=B_{1}\left(D \cap D_{1}\right)$. Thus (iv) holds and the lemma is proved.

We are now in a position to begin to pin things down very tightly.

Lemma 4.7. The following conditions hold:

(i) If $D \in D$ and $J^{*}(S) \subseteq D$, then $D$ is elementary abelian and $D \in$ $M(S)$; and

(ii) If $D \in D$ and $J^{*}(S) \subseteq D$, then $D=S$.

Proof. Assume $J^{*}(S) \nsubseteq \subseteq D$ and $D$ is not elementary abelian. Choose $D_{1} \in D$ as in Lemma 4.5. By Lemma 4.6(iv), we have $D=\left(D \cap D_{1}\right) B$. Since $B \subseteq Z=\Omega_{1}(Z(D))$, it follows that $F=\mho^{1}\left(D \cap D_{1}\right) \neq 1$. But then $F \subseteq$ $\mho^{1}(D) \cap \mho^{1}\left(D_{1}\right)$, whence by Lemma $2.1,\left\langle N^{*}, N_{1}^{*}\right\rangle \subseteq N_{G}(F)$. Hence by Lemma 4.4, $N_{1}^{*} \subseteq N_{G}(F)^{*} \subseteq N_{G}(B)$. But then $N_{1}^{*}$ normalizes $B D_{1}$. Since $B D_{1}=S$ by Lemma $4.6(\mathrm{i})$, this contradicts the fact that $\bar{N}_{1}^{*} \cong L_{2}\left(2^{n}\right)$ with $n \geqslant 1$. We thus conclude that $D$ is elementary abelian. By symmetry $D_{1}$ is also elementary abelian.

Next, as noted in the beginning of Lemma 4.5, $(A \cap D) Z \in M(S)$. However, $Z=D$ as $D$ is elementary abelian. Thus $(A \cap D) Z=D \in M(S)$, In particular, (i) holds.

Finally, as $D$ and $D_{1} \in M(S), J^{*}(S)=S$ by definition of $J^{*}(S)$. But now (ii) is clear.

Now choose $D$ and $D_{1}$ as in Lemmas 4.1 and 4.5. Set $Y=N_{G}(S)$.

LEMMA 4.8. The following conditions hold:

(i) $D \cap D_{1}=Z(S)$;

(ii) $M(S)=N(S)=\left\{D, D_{1}\right\}$ and $I(S)=D^{\#} \cup D_{1}^{\#}$;

(iii) If $A$ is a maximal abelian subgroup of $S$, then $A=D, A=D_{1}$ or $\Omega_{1}(A)=Z(S)$

(iv) If $X$ is a nonabelian subgroup of $S$, then $Z(X) \subseteq Z(S)$; and

(v) $D=\left\{D, D_{1}, S\right\}$ and $Y \subseteq N \cap N_{1}$. 
Proof. We know from the preceding lemmas that $S=D D_{1}$ with $D$ and $D_{1} \in M(S)$. Clearly then (i) holds. We argue next that $\left[d, d_{1}\right] \neq 1$ for any $d \in D-Z(S)$ and $d_{1} \in D_{1}-Z(S)$. Indeed, as $B$ covers $D / D \cap D_{1}=D / Z(S)$, $d=b z$ with $b \in B, z \in Z(S)$. Since $d \notin Z(S), b \in B-Z(S)$. In particular $b \notin B_{0}[B, S]$, and since $B / B_{0}$ is a standard $\bar{N}^{*}$-module, the assumption $d_{1} \notin$ $D$ implies $1 \neq\left[b, d_{1}\right]=\left[d, d_{1}\right]$.

In particular, it follows that $D$ and $D_{1}$ are maximal abelian subgroups of $S$. Since $S=B D_{1}$, with $D=B Z(S)$, this also implies that no element of $S-$ $\left(D \cup D_{1}\right)$ is an involution. This immediately yields (ii). Likewise (iii) and (iv) follow from these conditions. Furthermore, Lemma 4.7 implies that any element of $D$ other than $S$ is actually an element of $M(S)$. We conclude therefore from (ii) that $D=\left\{D, D_{1}, S\right\}$. Finally (ii) clearly implies that $Y$ leaves both $D$ and $D_{1}$ invariant, whence $Y \subseteq N \cap N_{1}$. Thus (v) also holds.

Now define $F=C_{D}\left(N^{*}\right)$ and $F_{1}=C_{D_{1}}\left(N_{1}^{*}\right)$. Clearly then $F$ and $F_{1}$ lie in $Z(S)$.

LEMMA 4.9. The following conditions hold:

(i) $D=F B$ and $D_{1}=F_{1} B_{1}$;

(ii) $F F_{1} \subseteq Z(S)$ and $F \cap F_{1}=1$;

(iii) $|F|=\left|F_{1}\right| \leqslant 2^{n}=|S: D|$; and

(iv) $n \geqslant 2$.

Proof. As $D$ and $D_{1}$ are elementary abelian, (i) follows from Lemma 4.2(v). We have already noted that $F F_{1} \subseteq Z(S)$. Furthermore, as $Y \subseteq N \cap$ $N_{1}, Y$ leaves both $F$ and $F_{1}$ invariant. Since $N=N^{*} Y$ and $N_{1}=N_{1}^{*} Y$, this implies that $F \cap F_{1}$ is invariant under $N, N_{1}$, and $Y$. Since $D=\left\{D, D_{1}\right.$, $S$ \}, we conclude that $F \cap F_{1}$ is strongly closed in $S$ with respect to $G$. As $G$ is a restricted simple group, $F \cap F_{1}$ must be trivial. Thus (ii) also holds.

From the structure of $D$, we have $|D: F|=|B:(B \cap F)|=2^{2 n}$, whence $|S: F|=2^{3 n}$ and likewise $\left|S: F_{1}\right|=2^{3 n}$. In particular, $|F|=\left|F_{1}\right|$. Since $Z(S)=D \cap D_{1}$, we know that $|S: Z(S)|=2^{2 n}$, and (ii) implies $|S|=$ $2^{2 n}|Z(S)| \geqslant 2^{2 n}|F|^{2}$. Since $|S: F|=2^{3 n}$, it follows that $|S|=2^{3 n}|F|$ and we conclude that $|F| \leqslant 2^{n}$. This establishes (iii).

Finally suppose that $n=1$, in which case $|S: D|=2$ and $D=F \times B$ by Lemma 4.2 (vi). $B$ is a standard $L_{2}$ (2)-module, so $|B|=4$ and $|S|=8$ or 16. However, $S \mathrm{SN}_{3}(S)$ is nonempty and $S$ is nonabelian as $G$ is restricted. Thus, in fact, $|S|=16$. Choosing $b \in B-Z(S), b_{1} \in B_{1}-Z(S)$, we see that $Q=\left\langle b, b_{1}\right\rangle \cong D_{8}$ and $S=Q Z(S)$. This forces $S=Q \times E$, where $E \cong Z_{2}$. Thus $S \cong D_{8} \times E_{2}$, which is impossible by [I, (2.74)] as $G$ is simple. We must have $n \geqslant 2$.

We complete this section by treating the case $F=1$. 
Proposition 4.10. If $F=1$, then $G \cong L_{3}\left(2^{n}\right)$.

Proof. In this case, $D=Z \cong E_{2} 2 n$ and $\bar{N}^{*}$ acts faithfully and irreducibly on $Z$. Since $S$ splits over $D$ by Lemma 4.2(ii), it follows from Gaschütz' theorem that $N^{*}$ splits over $Z$. But now it is immediate that $S$ is isomorphic to a Sylow 2-subgroup of $L_{3}\left(2^{n}\right)$ as $Z$ is a natural $\bar{N}^{*}$-module. Since $n \geqslant 2$, Proposition 3.1 now yields that $G \cong L_{3}\left(2^{n}\right)$.

5. A characterization of the groups $P S p\left(4,2^{n}\right)$. We continue the analysis of the preceding section, preserving the same notation. In view of Proposition 4.10 , it remains to treat the case $F \neq 1$. We shall prove

Proposition 5.1. If $F \neq 1$, then $G \cong \operatorname{PSp}\left(4,2^{n}\right)$.

Together Propositions 4.10 and 5.1 will establish Theorem B. We argue in a sequence of lemmas.

Since $n \geqslant 2, \bar{N}^{*}$ and $\bar{N}_{1}^{*}$ are simple groups. Let $\bar{R}$ be a complement to $\bar{S}$ in $N_{\bar{N}^{*}}(\bar{S})$. Clearly we can find a subgroup $R \cong Z_{2^{n}-1}$ in $Y$ such that $R$ projects isomorphically onto $\bar{R}$. Define $\bar{R}_{1}$ and $R_{1}$ similarly. Let $\tilde{Y}=Y / S$.

LEMMA 5.2. The following conditions hold:

(i) $F \cong F_{1} \cong E_{2^{n}}$;

(ii) $D \cong D_{1} \cong E_{3^{n}}$;

(iii) $Z(S)=F \times F_{1}$ and $|S|=2^{4 n}$;

(iv) $\widetilde{R}$ and $\widetilde{R}_{1}$ are normal in $\widetilde{Y}$ and $\widetilde{R} \widetilde{R}_{1}=\widetilde{R} \times \widetilde{R}_{1} \cong Z_{2^{n}-1} \times Z_{2^{n}-1}$;

(v) $R$ acts regularly on $F_{1}$ and $R_{1}$ acts regularly on $F$;

(vi) $\overline{R N}_{1}^{*} \cong \bar{R}_{1} \bar{N}^{*} \cong G L\left(2,2^{n}\right)$.

Proof. We know that $|S|=2^{n}|D|=2^{3 n}|F|$. Thus (i) and (ii) will follow from the known structure of $S$ and the symmetry between $D$ and $D_{1}$ once we show $|F|=2^{n}$. We have $R_{1} \subseteq Y \subseteq N$ and $F=C_{D}\left(N^{*}\right)$ is normal in $N$. Thus $R_{1}$ normalizes $F$. As $B_{1}$ is a standard $\bar{N}_{1}^{*}$-module, $R_{1}$ acts regularly on $\left(B_{1} \cap Z(S)\right) / B_{1} \cap F_{1}$. However, by Lemma 4.9(ii), $F F_{1} \subseteq Z(S)$, and we know from the structure of $D_{1}$ that $Z(S)=\left(B_{1} \cap Z(S)\right) F_{1}$. Thus $R_{1}$ acts regularly on $Z(S) / F_{1}$. As $F$ is $R_{1}$-invariant and $F \cap F_{1}=1$, again by Lemma 4.9 (ii) $R_{1}$ must act regularly on $F$. Since $F \neq 1$ by hypothesis, this forces $|F| \geqslant 2^{n}$, whence $|F|=2^{n}$ by Lemma 4.9 (iii). To prove (iii), we note that by Lemma 4.8(i) $Z(S)=D \cap D_{1}$, whence $|Z(S)|=2^{2 n}$. Since $F F_{1} \subseteq Z(S)$ and $F \cap F_{1}=1$, this yields $Z(S)=F_{1} \times F$.

We know that $R_{1}$ acts trivially on $F_{1}$ and regularly on $F$, and by 
symmetry $R$ acts trivially on $F$ and regularly on $F_{1}$. In particular, (v) holds. Certainly then $\widetilde{R} \cap \widetilde{R}_{1}=1$. Further, $Y \subseteq N$ implies that $\bar{Y}$ normalizes $N_{\bar{N}^{*}}(\bar{S})=\overline{R S}$, whence $\widetilde{Y}$ normalizes $\widetilde{R}$. By symmetry, $\widetilde{Y}$ normalizes $\widetilde{R}_{1}$. Since $\widetilde{R} \cap \widetilde{R}_{1}=1$ and $\widetilde{R} \cong \widetilde{R}_{1} \cong Z_{2^{n}-1}$, we see that (iv) holds.

Finally let $X$ be a subgroup of $Y$ of odd order which maps on $\widetilde{R} \widetilde{R_{1}}$, so that $X \cong Z_{2^{n}-1} \times Z_{2^{n}-1}$ and $\overline{X N}^{*}=\bar{R}_{1} \bar{N}^{*}$ with $\bar{X} \cap \bar{N}^{*} \cong Z_{2^{n}-1}$ and normalizing $\bar{S}$. Considering the structure of $\bar{X}$ and applying [I, (2.20)], we conclude that $\overline{X N}^{*}=\bar{R}_{1} \bar{N}^{*} \cong G L\left(2,2^{n}\right)$. By symmetry, also $\overline{R N}^{*} \cong G L\left(2,2^{n}\right)$.

We need one further fact

Lemma 5.3. We have $N_{G}(Z(S)) \subseteq Y$.

Proof. Let $H=N_{G}(Z(S))$ and $P=O_{2}(H)$. By the hypothesis of Theorem $\mathrm{B}, H$ is 2-constrained and has trivial core. Clearly $S \subseteq H$, and as $S / Z(S)$ is abelian, $S$ centralizes $P / Z(S)$ and $Z(S)$. Since $P$ and $Z(S)$ are $H$-invariant, $H^{*}$ centralizes $P / Z(S)$ and $Z(S)$, whence $O^{2}\left(H^{*}\right)$ centralizes $P$. Thus $O^{2}\left(H^{*}\right) \subseteq P$ and it follows that $H^{*}=P$, whence $P=S$ and we are done. Now we can prove

LEMMA 5.4. The following conditions hold:

(i) $N$ and $N_{1}$ are maximal 2-local subgroups of $G$ and are the only maximal 2-local subgroups containing $S$;

(ii) $N \cap N_{1}=Y$;

(iii) $N$ is the unique maximal 2-local subgroup of $G$ containing $N^{*}$ and $N_{1}$ is the unique maximal 2-local subgroup containing $N_{1}^{*}$.

Proof. Let $H$ be an arbitrary 2-local subgroup of $G$ containing $S$, and let $Q=O_{2}(H)$. We know that $O(H)=1$ and $C_{H}(Q) \subseteq Q$. If $Q \subseteq D$ or $D_{1}$, correspondingly $Q=D$ or $D_{1}$ as $D$ and $D_{1}$ are abelian. Hence in this case, $H \subseteq N$ or $N_{1}$. If $Q \nsubseteq D$ and $Q \nsubseteq D_{1}$, then by Lemma 4.8(iii) and (iv), $\Omega_{1}(Q)=Z(S)$ or $Z(Q) \subseteq Z(S)$. As $Z(S) \subseteq Z(Q), Z(Q)=Z(S)$ in the latter case and in both cases $Z(S)$ is characteristic in $Q$. Hence $H \subseteq N_{G}(Z(S))$ and so $H \subseteq Y$ by the preceding lemma. Since $Y \subseteq N \cap N_{1}$ by Lemma 4.8(v), $H \subseteq N$ or $N_{1}$ in this case as well. Thus (i) holds.

By Lemma 4.6(i), $S=D D_{1}$. Thus $N \cap N_{1} \subseteq N_{G}\left(D D_{1}\right)=N_{G}(S)=Y$.

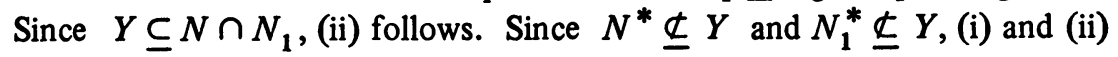
together immediately yield (iii).

REMARK. At this point we know that for $z$ in $F^{\#}, C_{G}(z)$ contains a normal subgroup of odd index isomorphic to the centralizer of the corresponding involution in $\operatorname{PSp}\left(4,2^{n}\right)$. Undoubtedly Suzuki's announced centralizer of involu- 
tion characterization of the groups $\operatorname{PSp}\left(m, 2^{n}\right)$ can be modified to cover the case of odd order extensions. However, it is easy enough to complete the characterization of $P s p\left(4,2^{n}\right)$ directly from the information we have established.

LemMa 5.5. The following conditions hold:

(i) $S^{\prime}=Z(S)=F \times F_{1}$;

(ii) $B=D$ and $B_{1}=D_{1}$;

(iii) $G$ has three conjugacy classes of involutions, each of which is central;

(iv) The elements of $F^{\#}, F_{1}^{\#}$ and $Z(S)-\left(F \cup F_{1}\right)$ constitute the distinct conjugacy classes of involutions of $Z(S)$;

(v) If $A$ and $A_{1}$ denote $X$-invariant complements to $Z(S)$ in $D$ and $D_{1}$ respectively, then
(a) $A^{\#} \sim F_{1}^{\#}$ and $F A-(F \cup A) \sim Z(S)-\left(F \cup F_{1}\right)$ in $N$;
(b) $A_{1}^{\#} \sim F^{\#}$ and $F_{1} A_{1}-\left(F_{1} \cup A_{1}\right) \sim Z(S)-\left(F \cup F_{1}\right)$ in $N_{1}$.

Proof. By Lemma 5.2(iii), we have $Z(S)=F \times F_{1}$. Furthermore, we have shown in Lemma 5.2 that $F$ is $R_{1}$-invariant, so $F$ is $X$-invariant and similarly $F_{1}$ is $X$-invariant. Moreover, we know that $R, R_{1}$ centralize $F, F_{1}$, respectively, and that $R_{1}, R$ act regularly and transitively on $F, F_{1}$, respectively. These conditions imply that $F$ and $F_{1}$ are nonisomorphic as $X$-modules and that they are the only nontrivial proper $X$-invariant subgroups of $Z(S)$. Since $S^{\prime} \neq 1$ and $S^{\prime}$ is an $X$-invariant subgroup of $Z(S)$, either (i) holds or else $S^{\prime}$ $=F$ or $F_{1}$. Consider the latter case and suppose, for definiteness, that $S^{\prime}=F$.

By Lemma 4.3, $J$ and hence $\bar{N}^{*}=\bar{J}$ acts indecomposably on $B$ and $B=$ $\left[B, \bar{N}^{*}\right]$. We apply Lemma 2.9 to the semidirect product $B\left(\bar{X} \bar{N}^{*}\right)$. Moreover, we see that the assumptions of Lemma 2.9 are satisfied. We therefore conclude that $m(B)=2 n$ or $3 n$ and correspondingly that $(B \bar{S})^{\prime}$ is of rank $n$ or $2 n$. However, the latter case is excluded here as $S^{\prime}=F$ is of rank $n$ under our present assumption. Thus $m(B)=2 n$ and $B$ is a standard $\bar{N}^{*}$-module. In particular, $R$ acts regularly on $B$. However, it also follows that $B \cap S^{\prime} \neq 1$ and is $X$-invariant, whence $B \cap S^{\prime}$ $=F$, contrary to the fact that $R$ acts trivially on $F$ and $F \neq 1$. Thus (i) holds. Since $\bar{N}^{*}$ centralizes $D / B$, we see that $S / B$ is abelian, whence $S^{\prime} \subseteq B$. Hence $F \subseteq$ $B$ by (i). Now our conditions force $m(B)=3 n$. We conclude therefore from Lemma 5.2(ii) that $B=D$. Similarly $B_{1}=D_{1}$, so (ii) also holds.

By Lemma 2.9(iv), we have that all involutions of $N$ and hence of $G$ are central. Observe next that $F=C_{Z}(J), \bar{J}=\bar{N}^{*} \triangleleft \bar{N}$ and $N_{G}(S) \subseteq N$, so $N_{G}(S)$ leaves $F$ invariant. Similarly $N_{G}(S)$ leaves $F_{1}$ invariant and so also leaves $F_{2}=Z(S)-\left(F \cup F_{1}\right)$ invariant. But we know that $X \subseteq N_{G}(S)$ acts transitively on $F^{\#}$ and $F_{1}^{\#}$. Moreover, if we use Lemma 5.2, we easily obtain that $X$ 
acts transitively on $F_{2}=F_{2}^{\#}$. On the other hand, any two involutions of $Z(S)$ conjugate in $G$ are necessarily conjugate in $N_{G}(S)$. Hence no elements from two of the sets $F^{\#}, F_{1}^{\#}, F_{2}^{\#}$ can be conjugate in $G$ and so (iii) and (iv) hold. Finally as $\overline{X J}=\overline{X N}^{*} \cong G L\left(2,2^{n}\right)$ and $\bar{X}$ is a complement to $\bar{S}$ in $N_{\overline{X N}}(\bar{S})$, it is immediate that $F, F_{1}$, and $A$ are nonisomorphic $\bar{X}$-modules with $\bar{R}$ acting trivially on $F$ and nontrivially on $F_{1}$ and $A$. But $\bar{N}^{*}$ contains an involution $\bar{w}$ which normalizes $\bar{X}$ and inverts $\bar{X} \cap \bar{N}^{*}$. These conditions clearly force $\bar{w}$ to interchange $F_{1}$ and $A$, whence $F_{1}^{\#} \sim A^{\#}$ in $N$. Since $\bar{w}$ centralizes $F$, it also transforms $F A-(F \cup A)$ into $Z(S)-(F \cup$ $\left.F_{1}\right)$. Thus (v)(a) holds and similarly we obtain (v)(b).

As to be expected, the next step in the analysis is to demonstrate that the group $\left\langle X, N^{*}, N_{1}^{*}\right\rangle$ is a split $(B, N)$-pair isomorphic to $\operatorname{PSp}\left(4,2^{n}\right)$. To accomplish this, it remains only to determine its Weyl group. To this end, we introduce some further notation. We let $V, V_{1}$ be cyclic subgroups of $X$ of order $2^{n}-1$ such that $\bar{V}$ centralizes $\bar{N}^{*}$ and $\bar{V}_{1}$ centralizes $\bar{N}_{1}^{*}$. Setting $K=C_{N^{*}}(V)$, it follows that $K$ covers $\bar{N}^{*}$. Since $\bar{N}^{*}$ acts indecomposably on $D$ by Lemma 4.3 , and since $\bar{R}=C_{\bar{X}}(\bar{F})$ implies $V$ acts regularly on $F$, it is immediate that $C_{D}(V)=1$. Hence $K \cong L_{2}\left(2^{n}\right)$ and consequently $V K \cong$ $G L\left(2,2^{n}\right)$. Similarly if we set $K_{1}=C_{N_{1}^{*}}\left(V_{1}\right)$, we obtain that $K_{1} \cong L_{2}\left(2^{n}\right)$ and $V_{1} K_{1} \cong G L\left(2,2^{n}\right)$.

Next we set $U=C_{S}(V)$ : Since $\bar{V}$ centralizes $\bar{S}, U$ covers $S / D$ and as $C_{D}(V)=1$, it follows that $U \cong E_{2^{n}}$ and that $U \in S(K)$. Similarly $U_{1}=$ $C_{S}\left(V_{1}\right) \cong E_{2^{n}}$ and $U_{1} \in S\left(K_{1}\right)$. Furthermore, as $U \cap D=1$, we have that $U \subseteq D_{1}-Z(S)$ by Lemma 4.8(ii) and similarly $U_{1} \subseteq D-Z(S)$. But we know that $X$ is abelian and so $X$ leaves both $U$ and $U_{1}$ invariant. Thus $Z=D$ $=Z(S) \times U_{1}$ and $Z_{1}=D_{1}=Z(S) \times U$. Lemma $5.5(\mathrm{v})$ implies that $U_{1}^{\#} \sim$ $F_{1}^{\#}$ in $N$, and $U^{\#} \sim F^{\#}$ in $N_{1}$. Now Lemma 5.5(iv) yields the important conclusion that no element of $U^{\#}$ is conjugate in $G$ to an element of $U_{1}^{\#}$.

Finally by the structure of $X K$, we know that $N_{K}(X)$ contains an involution $w$ and that $U \cap U^{w}=1$. Similarly $N_{K_{1}}(X)$ contains an involution $w_{1}$ and $U_{1} \cap U_{1}^{w}{ }^{1}=1$. We must determine the order of the group $\left\langle w, w_{1}\right\rangle$. We shall prove

Lemma 5.6. The group $\left\langle w, w_{1}\right\rangle$ is dihedral of order 8 .

Proof. Since $w$ and $w_{1}$ are involutions, the lemma will follow provided we show that $w w_{1}$ has order 4 . We claim first that $\left|w w_{1}\right|$ is even; so assume 
false, in which case $w \sim w_{1}$ in $\left\langle w, w_{1}\right\rangle$. But as $U \in S(K)$ and $K$ has only one class of involutions, $w \sim u$ in $K$ for some $u$ in $U^{\#}$. Similarly $w_{1} \sim u_{1}$ in $K_{1}$ for some $u_{1}$ in $U_{1}^{\#}$. But then $u \sim u_{1}$ in $G$, contrary to what we have shown above. This proves our assertion.

Our argument yields that $\left\langle w w_{1}\right\rangle$ contains a unique involution $z$. Then $w$ and $w_{1}$ both lie in $H=C_{G}(z)$. By Lemma 5.5(iii), $z$ is a central involution, whence $S^{g} \subseteq H$ for some $g$ in $G$. But $H$ is a 2-local subgroup and consequently Lemma 5.4(i) yields that $H \subseteq N^{g}$ or $N_{1}^{g}$. By symmetry, we can assume, for definiteness, that $H \subseteq N^{g}$.

We use this to prove that $\left|w w_{1}\right|=2^{a}$ for some $a$; so suppose false and let $y$ be an element of odd prime order $p$ in $\left\langle w w_{1}\right\rangle$. By the structure of $N$, we have that $N^{*}=D K$ and consequently $\left\langle w, w_{1}\right\rangle \subseteq(D K)^{g}$. Since $K^{g}$ contains a Sylow $p$-subgroup of $(D K)^{g}$, it follows that $\overline{y^{d}} \in K^{g}$ for some $d$ in $D^{g}$. We have that $w^{d}$ and $w_{1}^{d}$ invert $y^{d}$ and so lie in the extended centralizer $C$ of $y^{d}$ in $N^{g}=(V K D)^{g}$. Since $K^{g} \cong L_{2}\left(2,2^{n}\right), C_{K^{g}}\left(y^{d}\right)=P$ is cyclic of order $2^{n}-1$ or $2^{n}+1$ and $C \cap K^{g}=P\langle t\rangle$ for some involution $t$ of $K^{g}$ which inverts $y^{d}$. We also have that $V^{g}$ centralizes $y^{d}$. Furthermore, it is immediate from the action of $K^{g}$ on $D^{g}$ that $y^{d}$ acts regularly on $D^{g} / F^{g}$ and consequently $C_{D^{g}}\left(y^{d}\right)=F^{g}$. We thus conclude that

$$
C=V^{g}\left(F^{g} \times P\langle t\rangle\right),
$$

with $V^{g}$ acting transitively on $\left(F^{g}\right)^{\#}$ and centralizing $P\langle t\rangle$.

But now we see that $Q=F^{g}\langle t\rangle \cong E_{2^{n+1}}$ is a Sylow 2-subgroup of $C$ and that $V^{g}$ fixes $t$ and acts transitively on the remaining involutions of $Q-$ $F^{g}$. Thus $C-F^{g}$ has exactly two conjugacy classes of involutions. Since $w^{d}$ and $w_{1}^{d}$ invert $y^{d}$, clearly both lie in $C-F^{g}$ and as these two involutions are not conjugate in $G$, they are not conjugate in $C$. We conclude that one of the elements $w$ or $w_{1}$ is conjugate in $G$ to $t$ and the other to $f t$, where $f \in$ $F^{g}$ and $f \neq 1$. But $t$ is conjugate in $G$ to an involution of $K$ and hence to $w$, so, in fact, we have $w_{1} \sim f t$ in $G$. On the other hand, as $F^{g} K^{g}=$ $(F \times K)^{g}, f t \sim f_{0} u_{0}$ in $G$ for suitable $f_{0} \in F^{\#}$ and $u_{0} \in U^{\#}$. But now it follows from Lemma 5.5(v) that $f_{0} u_{0}$ and hence $w_{1}$ is conjugate in $G$ to an element of $Z(S)-\left(F \cup F_{1}\right)$. However, $w_{1}$ is conjugate in $K_{1}$ to an element of $U_{1}^{\#}$ and so to an element of $F^{\#}$ by the same lemma. Clearly this contradicts Lemma 5.5(iv). Thus $\left|w w_{1}\right|=2^{a}$ for some $a$, as asserted.

If $a \geqslant 3$, then $w, w_{1}$ would have class at least 3 , contrary to the fact that $S$ has class 2 . Hence $a \leqslant 2$ and so to complete the proof, it remains only to prove equality. 
Suppose then that $a=0$ or 1 , in which cases $w$ centralizes $w_{1}$. Clearly $K \subseteq N^{*}$ and so $K$ centralizes $F$ by definition of $F$. Hence $w$ centralizes $F$. Since $w_{1} \in K_{1}$ normalizes $D_{1}$, it follows therefore that $F^{w w_{1}}=F^{w_{1}} \subseteq D_{1}$. Likewise, $F^{w_{1}} \subseteq D_{1}$ and so $F^{w_{1} w} \subseteq D_{1}^{w}$. Since $w w_{1}=w_{1} w$, we conclude that $F^{w w_{1}} \subseteq \bar{D}_{1} \cap D_{1}^{w}$. However, as the Sylow 2-subgroups of $\bar{N}=N / D$ are disjoint from each other, $D_{1} \cap D_{1}^{w} \subseteq D=O_{2}(N)$ and consequently $D_{1} \cap D_{1}^{w}$ $\subseteq D_{1} \cap D=Z(S)$. Thus $F$ and $F^{w w_{1}}$ are subgroups of $Z(S)$ that are conjugate in $G$ and so they are conjugate in $Y=N_{G}(S)$. But $Y \subset N$ by Lemma 4.8(v), whence $Y$ normalizes $F=C_{D}\left(N^{*}\right)$ and so $F=F^{w w_{1}}$. Thus $w w_{1} \in$ $N_{G}(F)$. But $N_{G}(F) \subseteq N$ by Lemma 5.4. Hence $w w_{1} \in N$ and therefore $w_{1}$ $\in N$. Since $U_{1} \subseteq D \triangleleft N$, this implies that $\left\langle U_{1}, w_{1}\right\rangle$ is a 2-group. However, this is impossible as $U_{1} \cap U_{1}^{w}=1$ and $U_{1}$ is a Sylow 2-subgroup of $K_{1}$.

Now we obtain our objective.

Lemma 5.7. The group $\left\langle X, N, N_{1}^{*}\right\rangle$ is a split $(B, N)$-pair isomorphic to $\operatorname{Psp}\left(4,2^{n}\right)$.

Proof. Each of the groups $U, U_{1}, F$, and $F_{1}$ is $X$-invariant (they are the "root groups" of $S$ ). Moreover, we see that $D=U_{1} \times F \times F_{1}$ and $D_{1}=U \times$ $F \times F_{1}$, while $S=U U_{1} F F_{1}$. Since $\bar{K}=\bar{N}^{*}$, we also have that $\bar{w}$ inverts $\bar{R}$ and so $w$ leaves $B=[D, \bar{R}]$ invariant. Clearly $B=U_{1} \times F_{1}$ and our conditions imply that $U_{1}, F_{1}$ are the only two irreducible $X$-submodules of $B$. Since $\bar{w}$ inverts $\bar{R}, w$ must interchange $U_{1}$ and $F_{1}$. We know that $w$ centralizes $F$. Similarly, $w_{1}$ interchanges $U$ and $F$ and centralizes $F_{1}$. Thus we have the following table for the action of $w$ and $w_{1}$ on the root groups of $S$ :

\begin{tabular}{c|c|c} 
& $w$ & $w_{1}$ \\
\hline$U$ & - & $F$ \\
$U_{1}$ & $F_{1}$ & - \\
$F$ & $F$ & $U$ \\
$F_{1}$ & $U_{1}$ & $F_{1}$
\end{tabular}

Furthermore, if we set $w_{0}=\left(w w_{1}\right)^{2}$, we claim that $S \cap S^{w_{0}}=1$.

To prove this, observe first that $w_{0}$ does not lie in $N$ or $N_{1}$. Indeed, suppose false. Since $w_{0}=\left(w w_{1}\right)^{2}=\left(w_{1} w\right)^{2}$, we can assume, by symmetry, that $w_{0} \in N$, whence $D^{w_{0}}=D$. Since $w$ normalizes $D$, this yields $D=$ $D^{w}{ }^{w} w_{1} \supset F_{1}^{w} w^{w w_{1}}=U_{1}^{w}$. But then $\left\langle U_{1}, U_{1}^{w}\right\rangle$ is a 2-group, which is not 
the case. This proves our assertion. This in turn implies that $w_{0}$ normalizes no nontrivial subgroup $Z_{0}$ of $Z(S)$; otherwise $w_{0} \in N_{G}\left(Z_{0}\right) \subseteq N$ or $N_{1}$ by Lemma 5.4(i).

Suppose now that $S_{0}=S \cap S^{w_{0}} \neq 1$. Since $w_{0}$ is an involution, $w$ normalizes $S_{0}$. However, $\mho^{1}\left(S_{0}\right) \subseteq Z(S)$, and so by the preceding paragraph $S_{0}$ must be elementary abelian, otherwise $\mho^{1}\left(S_{0}\right)$ would be a nontrivial subgroup of $Z(S)$ invariant under $w_{0}$. Thus $S_{0} \subseteq D$ or $D_{1}$. But $S_{0}$ is $X$-invariant as $S$ is $X$-invariant and $X$ is $w_{0}$-invariant. However, it is immediate that $U_{1}, F$, and $F_{1}$ are the only minimal $X$-invariant subgroups of $D_{1}$. Hence by the preceding paragraph, we have that $S_{0} \supseteq U$ or $U_{1}$. By symmetry, we can suppose that $S_{0} \supseteq U_{1}$. Since $U_{1}^{w}=F_{1}$, this yields $F_{1}^{w} 1^{w w_{1}}=U_{1}^{w} 0 \subset D$, giving the same contradiction as above. Thus $S_{0}=1$, is asserted.

But now setting $B=X S$ and $N=X\left\langle w, w_{1}\right\rangle$ (there will be no confusion here with our previous uses of $B$ and $N$ ), we conclude now from a theorem of Tits [4] that the group $G_{0}=\langle B, N\rangle$ is a split $(B, N)$-pair with Weyl group $W=\left\langle w, w_{1}\right\rangle$. Since we also have $X N^{*}=\langle X, S, w\rangle$ and $X N_{1}^{*}=\left\langle X, S, w_{1}\right\rangle$, we see that $G_{0}=\left\langle X, N^{*}, N_{1}^{*}\right\rangle$. Finally as $W$ is dihedral of order 8 and $X \cong$ $Z_{2^{n}-1} \times Z_{2^{n}-1}$,Proposition 3.2 yields that $G_{0} \cong P S p\left(4,2^{n}\right)$ and the lemma is proved.

Now we can complete the proof of Proposition 5.1. We need only show that the group $G_{0}=\left\langle X, N^{*}, N_{1}^{*}\right\rangle$ of the preceding lemma is $G$ itself; so assume the contrary. Since $Y=N_{G}(S) \subseteq N \cap N_{1}, Y$ normalizes both $N^{*}$ and $N_{1}^{*}$. Moreover, by Lemma 5.2(iv), $S R$ and $S R_{1}$ are normal in $Y$, which implies that $Y$ also normalizes $S X$. Hence $Y$ normalizes $G_{0}$. Since $G$ is simple and $G_{0} \subset G$, it follows now that also $G_{1}=G_{0} Y \subset G$. But $N=N^{*} N_{N}(S)=N^{*} Y$ and so $N \subseteq G_{1}$. Similarly $N_{1} \subseteq G_{1}$. Since $D=\left\{D, D_{1}, S\right\}$, we see that $G_{1}$ contains the normalizer in $G$ of each element of $D$ and so $G_{1}$ controls fusion in $S$. Hence to prove that $G_{1}$ is strongly embedded in $G$, we need only show, in view of Lemma 5.5(iii), that $G_{1}$ contains $C_{G}(x)$ for $x$ in $Z(S)^{\#}$. However, as $S \subseteq C_{G}(x)$ for any such $x, C_{G}(x) \subseteq N$ or $N_{1}$ by Lemma 5.4(i) and so $C_{G}(x) \subseteq G_{1}$. We therefore conclude that $G_{1}$ is strongly embedded in $G$, which implies that $G$ has only one conjugacy class of involutions, contrary to Lemma 5.5 (iii), and the proposition is proved.

This completes the proof of Theorem B.

\section{BIBLIOGRAPHY}

1. M. Collins, The characterization of finite groups whose Sylow 2-subgroups are of type $L_{3}(q), q$ even, J. Algebra 25 (1973), 490-512. 
2. P. Fong and G. Seitz, The classification of split (B, N)-pairs of rank 2, Invent. Math. (to appear).

3. Z. Janko, $A$ new finite simple group with abelian Sylow 2-subgroups and its characterization, J. Algebra 3 (1966), 147-186. MR 33 \#1359.

4. J. Tits, Theorème de Bruhat et sous-groupes paraboliques, C. R. Acad. Sci. Paris 254 (1962), 2910-2912. MR 25 \#2149.

DEPARTMENT OF MATHEMATICS, STEVENS INSTITUTE OF TECHNOLOGY, HOBOKEN, NEW JERSEY 07030

DEPARTMENT OF MATHEMATICS, RUTGERS UNIVERSITY, NEW BRUNSWICK, NEW JERSEY 08903 